ESAIM: COCV 27 (2021) 65

https://doi.org/10.1051/cocv/2021059
ESAIM: Control, Optimisation and Calculus of Variations

www.esaim-cocv.org

\title{
FEEDBACK STABILIZATION OF A 3D FLUID FLOW BY SHAPE DEFORMATIONS OF AN OBSTACLE*
}

\author{
JeAn-Pierre Raymond ${ }^{1, * *}$ And Muthusamy VAnninathan ${ }^{2}$
}

\begin{abstract}
We consider a fluid flow in a time dependent domain $\Omega_{f}(t)=\Omega \backslash \overline{\Omega_{s}(t)} \subset \mathbb{R}^{3}$, surrounding a deformable obstacle $\Omega_{s}(t)$. We assume that the fluid flow satisfies the incompressible Navier-Stokes equations in $\Omega_{f}(t), t>0$. We prove that, for any arbitrary exponential decay rate $\omega>0$, if the initial condition of the fluid flow is small enough in some norm, the deformation of the boundary $\partial \Omega_{s}(t)$ can be chosen so that the fluid flow is stabilized to rest, and the obstacle to its initial shape and its initial location, with the exponential decay rate $\omega>0$.
\end{abstract}

Mathematics Subject Classification. 93B52, 93C20, 93D15, 35Q30, 76D55, 76D05, 74F10.

Received October 23, 2020. Accepted May 31, 2021.

Dedicated to Enrique Zuazua on the occasion of his $60^{\text {th }}$ birthday.

\section{INTRODUCTION}

\subsection{Setting of the problem}

We consider a fluid occupying a time dependent bounded domain $\Omega_{f}(t)$ in $\mathbb{R}^{3}$, for $t \geq 0$. We consider the case where $\Omega_{f}(t)=\Omega \backslash \overline{\Omega_{s}(t)}, \Omega$ is a regular bounded domain in $\mathbb{R}^{3}$, and $\Omega_{s}(t)$ is another regular domain such that $\overline{\Omega_{s}(t)} \subset \Omega$, for $t \geq 0$. Thus, the obstacle $\Omega_{s}(t)$ is surrounded by the fluid domain $\Omega_{f}(t)$ as in Figure 1 , but other situations can be considered as well. We denote by $\Gamma_{e}$ the boundary of $\Omega$, and by $\Gamma_{s}(t)$ the boundary of $\Omega_{s}(t)$. Therefore $\Gamma_{e} \cup \Gamma_{s}(t)=\partial \Omega_{f}(t), \Gamma_{s}(t)=\overline{\Omega_{f}(t)} \cap \overline{\Omega_{s}(t)}$, and $\overline{\Gamma_{s}(t)} \cap \overline{\Gamma_{e}}=\emptyset$. For simplicity, we assume that $\Gamma_{s}(t)$ is connected.

For $t=0$, we set $\Omega_{f}(0)=\Omega_{f}, \Omega_{s}(0)=\Omega_{s}, \Gamma_{s}(0)=\Gamma_{s}$.

Let $u(\cdot, t)$ and $p(\cdot, t)$ be the fluid velocity and pressure. We are going to consider the case when the fluid flow satisfies the incompressible Navier-Stokes equations in $\Omega_{f}(t)$, with no-slip boundary conditions at the boundary of $\Omega_{s}(t)$, and an initial condition $u(\cdot, 0)=u_{0} \in H_{0}^{1}\left(\Omega_{f}\right)$ satisfying $\operatorname{div}\left(u_{0}\right)=0$.

Our goal is to prove that, for any arbitrary exponential decay rate $\omega>0$, if $u_{0}$ is small enough in the norm $\|\cdot\|_{H^{1+\ell}\left(\Omega_{f}\right)}$ for some $\ell \in\left(\frac{1}{2}, \frac{3}{2}\right)$, it is possible to choose the shape of the obstacle $\Omega_{s}(t)$, or similarly the

* The authors are members of an IFCAM-project, Indo-French Centre for Applied Mathematics - UMI IFCAM, Bangalore, India, supported by DST - IISc - CNRS - and Université Paul Sabatier Toulouse III. The first author is supported by the ANR-project IFSMACS (ANR 15-CE40.0010).

Keywords and phrases: Deformable boundary, feedback control, stabilization, Navier-Stokes equations.

1 Institut de Mathématiques de Toulouse, UMR CNRS 5219, Université Paul Sabatier, 31062 Toulouse Cedex 9, France.

2 Department of Mathematics, Indian Institute of Technology, Bombay, Powai, Mumbai, Maharashtra 400076, India.

** Corresponding author: raymond@math.univ-toulouse.fr 

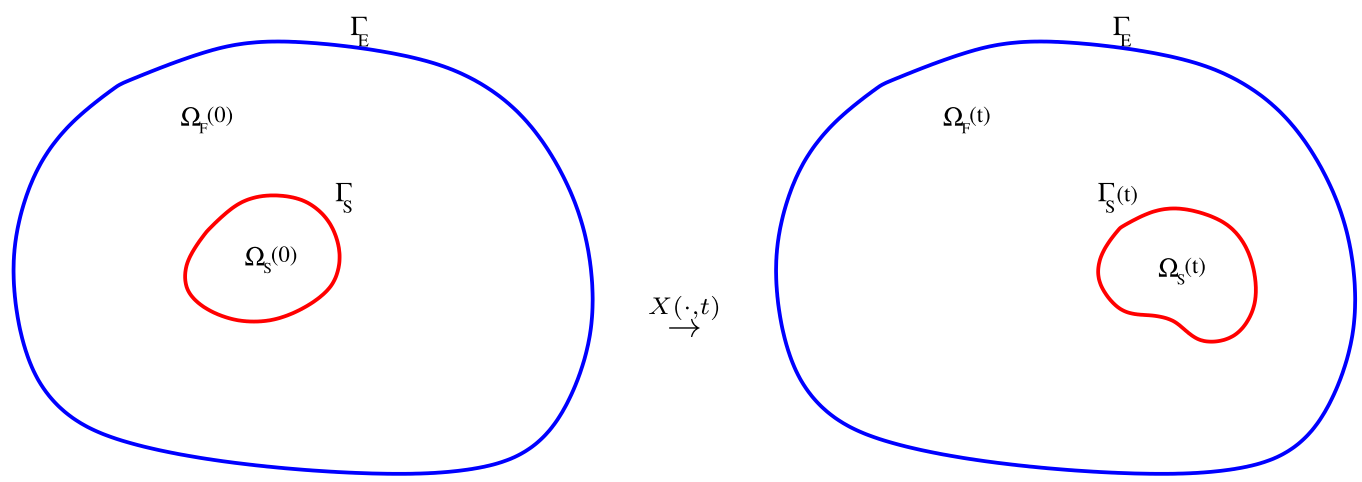

FIgURE 1. Reference and deformed configurations.

deformation of the boundary $\Gamma_{s}(t)$, for $t \geq 0$, in such a way that

$$
\lim _{t \rightarrow \infty}\left\|e^{\omega t} u(\cdot, t)\right\|_{H^{1+\ell}\left(\Omega_{f}(t) ; \mathbb{R}^{3}\right)}=0 .
$$

In order to deal with this nonlinear problem, we look for regular obstacle deformations $X_{s}(\cdot, t)$ : $\Omega_{s} \mapsto \Omega_{s}(t)$, or similarly, regular deformations of the fluid domain $X_{f}(\cdot, t): \Omega_{f} \mapsto \Omega_{f}(t)$. More precisely, we look for deformations which can be expressed as regular diffeomorphisms $X(\cdot, t)$ from $\overline{\Omega_{f}}$ into $\overline{\Omega_{f}(t)}$, or similarly from $\Gamma_{s}$ into $\Gamma_{s}(t)$.

Thus, we are led to consider the following system

$$
\begin{aligned}
& \frac{\partial u}{\partial t}+\left(u \cdot \nabla_{x}\right) u-\operatorname{div}_{x} \sigma(u, p)=0, \quad \operatorname{div}_{x} u=0 \quad \text { in } \Omega_{f}(t), \text { for } t>0, \\
& u=0 \quad \text { on } \quad \Sigma_{e}^{\infty}=\Gamma_{e} \times(0, \infty), \quad u(0)=u_{0} \text { in } \Omega_{f}, \\
& u(X(y, t), t)=\frac{\partial w}{\partial t}(y, t) \quad \text { for }(y, t) \in \Sigma_{s}^{\infty}=\Gamma_{s} \times(0, \infty), \\
& X(y, t)=y+w(y, t) \quad \text { for }(y, t) \in \Sigma_{s}^{\infty}, \quad X(y, 0)=y \quad \text { and } \quad w(y, 0)=0 \quad \text { for } y \in \Gamma_{s}, \\
& \Gamma_{s}(t)=X\left(\Gamma_{s}, t\right),
\end{aligned}
$$

where $u$ and $p$ are the fluid velocity and pressure, $w$ may be viewed as the obstacle displacement from the reference configuration $\Omega_{s}$,

$$
\sigma(u, p)=\nu\left(\nabla_{x} u+\left(\nabla_{x} u\right)^{T}\right)-p I,
$$

is the Cauchy stress tensor, $\nu>0$ is the fluid viscosity. Let us notice that, since $\operatorname{div}_{x} u=0$ in $\Omega_{f}(t)$, $-\operatorname{div}_{x} \sigma(u, p)=-\nu \Delta u+\nabla p$.

When $w$ satisfies the Lamé system of linear elasticity in $\Omega_{s} \times(0, \infty)$, coupling system (1.2) with the elasticity system (including the equality of the Cauchy stress forces at the interface $\Gamma_{s}$ ) corresponds to a fluid-structure interaction system (see, e.g., [25]). Thus there is a close connection between the stabilization of fluid-structure interaction systems and the stabilization problem that we consider. Here, for simplicity, we first treat the stabilization of the fluid flow by shape deformations of the surrounded obstable $\Omega_{s}(t)$. The stabilization of the system coupling the Navier-Stokes equations with the Lamé system will be studied in a forthcoming paper, based on the results obtained here. 
There are a few existence results of strong solutions, local in time, for system (1.2), either with a flat initial fluid-solid interface $[14,25]$, or in the case of the geometrical configuration considered here, see e.g. [3, 4]. The results in [16] and in [12] provide regularity results for strong solutions (assuming the existence of these strong solutions). Several papers are concerned with the case where the solid is rigid, we refer to [5, 11] and the references therein. There are other results dealing with models in which the fluid-solid interface is assumed to be fixed [14].

From the point of view of control theory a very few results are known for fluid-structure interaction models. We refer to the recent paper [28] where a stabilization result is obtained for a fluid-solid structure model, with a control acting in the solid equation. In [32, 33], the authors study the asymptotic behavior of fluid-structure models coupling heat and wave equations, but the systems are linear and the fluid-structure interface is not moving. In [18], the authors study some stabilization problems, but still in that case the fluid-structure interface is fixed. The case of a moving interface, with a stabilizing feedback located at the fluid-structure interface is considered in [13]. In [21], the second author has studied the stabilization of a fluid-structure model in two dimensions, where the structure is a damped beam equation located at the boundary of the fluid domain. These results have been recently extended to more complex systems, see [7]. We also mention [9, 10], where the shape of an elastic structure is used to reduce the drag of a fluid flow surrounding this an elastic structure.

We prove stabilization results for system (1.2) by rewriting it in Lagrangian variables. For that, we introduce

$$
\widetilde{u}(y, t)=u(X(y, t), t) \quad \text { and } \quad \widetilde{p}(y, t)=p(X(y, t), t),
$$

where

$$
\frac{\partial X}{\partial t}(y, t)=u(X(y, t), t), \quad X(y, 0)=y \quad \text { for all } y \in \overline{\Omega_{f}} .
$$

We notice that if $u$ satisfies $(1.2)_{3}$, then equation (1.3) restricted to $\Gamma_{s}$ reduces to

$$
\frac{\partial X}{\partial t}(y, t)=\frac{\partial w}{\partial t}(y, t) \quad \text { for all } y \in \Gamma_{s} .
$$

Since $w(\cdot, 0)=0$, this equation reduces to $(1.2)_{4}$. We look for $w$ of the form

$$
w(y, t)=\sum_{i=1}^{N_{c}} F_{i}(t) \xi_{i}(y) \quad \text { on } \Sigma_{s}^{\infty},
$$

where the functions $\left(\xi_{i}\right)_{1 \leq i \leq N_{c}}$ are regular and satisfiy

$$
\int_{\Gamma_{s}} \xi_{i} \cdot n \mathrm{~d} x=0 \quad \text { for all } 1 \leq i \leq N_{c}
$$

To ensure that the functions $\left(F_{i}\right)_{1 \leq i \leq N_{c}}$ are sufficiently regular, we are going to look for $\left(F_{i}\right)_{1 \leq i \leq N_{c}}$ satisfying

$$
\begin{array}{ll}
F_{i}^{\prime}(t)=f_{i}(t), & F_{i}(0)=0, \quad \text { for all } 1 \leq i \leq N_{c} \\
f_{i}^{\prime}(t)=h_{i}(t), & f_{i}(0)=0, \quad \text { for all } 1 \leq i \leq N_{c} .
\end{array}
$$


We set $F=\left(F_{1}, \ldots, F_{N_{c}}\right), f=\left(f_{1}, \ldots, f_{N_{c}}\right), h=\left(h_{1}, \ldots, h_{N_{c}}\right)$. The system satisfied by $(\widetilde{u}, \widetilde{p}, F, f)$ is of the form

$$
\begin{aligned}
& \frac{\partial \widetilde{u}}{\partial t}-\nu \Delta \widetilde{u}+\nabla \widetilde{p}=\mathcal{F}(\widetilde{u}, \widetilde{p}) \quad \text { and } \quad \operatorname{div} \widetilde{u}=\mathcal{G}(\widetilde{u})=\operatorname{div}(\mathbf{g}(\widetilde{u})) \quad \text { in } Q_{f}^{\infty}=\Omega_{f} \times(0, \infty), \\
& \widetilde{u}(0)=u_{0} \text { in } \Omega_{f}, \quad \widetilde{u}=0 \quad \text { on } \Sigma_{e}^{\infty}, \\
& \widetilde{u}(y, t)=\sum_{i=1}^{N_{c}} f_{i}(t) \xi_{i}(y) \quad \text { on } \Sigma_{s}^{\infty}, \\
& F^{\prime}(t)=f(t), \quad F(0)=0, \\
& f^{\prime}(t)=h(t), \quad f(0)=0, \\
& X(y, t)=y+\int_{0}^{t} \widetilde{u}(y, s) \mathrm{d} s, \quad \text { for all } y \in \Omega_{f} \text { and all } t \in[0, \infty), \\
& \Omega_{f}(t)=X\left(\Omega_{f}, t\right), \quad Y(X(y, t), t)=y \quad \text { for all } y \in \Omega_{f} \text { and all } t \in[0, \infty), \\
& \text { and } \quad X(Y(x, t), t)=x \quad \text { for all } x \in \Omega_{f}(t) \text { and all } t \in[0, \infty),
\end{aligned}
$$

where the nonlinear terms $\mathcal{F}, \mathcal{G}$, and $\mathbf{g}$ are defined by

$$
\begin{aligned}
& \mathcal{F}(\widetilde{u}, \widetilde{p})=\mathcal{F}_{1}(\widetilde{u})+\mathcal{F}_{2}(\widetilde{u})+\mathcal{F}_{3}(\widetilde{u}, \widetilde{p}), \\
& \mathcal{F}_{1}(\widetilde{u})=\nu \sum_{j, k} \frac{\partial^{2} Y_{k}}{\partial x_{j}^{2}}(X(y, t), t) \frac{\partial \widetilde{u}}{\partial y_{k}}(y, t), \\
& \mathcal{F}_{2}(\widetilde{u})=\nu \sum_{i, j, k} \frac{\partial Y_{i}}{\partial x_{j}} \frac{\partial Y_{k}}{\partial x_{j}}(X(y, t), t) \frac{\partial^{2} \widetilde{u}}{\partial y_{i} \partial y_{k}}(y, t)-\nu \Delta \widetilde{u}, \\
& \mathcal{F}_{3}(\widetilde{u}, \widetilde{p})=\left(I-J_{Y}^{T}\right) \nabla \widetilde{p}, \\
& \mathcal{G}(\widetilde{u})=\nabla \widetilde{u}:\left(I-\operatorname{det}\left(J_{X}\right) J_{Y}^{T}\right), \quad \text { and } \mathbf{g}(\widetilde{u})=\left(I-\operatorname{det}\left(J_{X}\right) J_{Y}\right) \widetilde{u} .
\end{aligned}
$$

In order to satisfy (1.1), we are going to look for a control $h \in L^{2}\left(0, \infty ; \mathbb{R}^{N_{c}}\right)$, in feedback form, such that the system (1.4) admits a unique solution $(\widetilde{u}, \widetilde{p}, F, f)$ satisfying

$$
\lim _{t \rightarrow \infty}\left\|e^{\omega t} \widetilde{u}(\cdot, t)\right\|_{H^{1+\ell}\left(\Omega_{f} ; \mathbb{R}^{3}\right)}=0
$$

In order to impose the exponential decay rate $\omega>0$, we introduce the following change of unknowns

$$
\begin{array}{ll}
\widehat{u}(y, t)=e^{\omega t} \widetilde{u}(y, t), & \widehat{p}(y, t)=e^{\omega t} \widetilde{p}(y, t), \quad \widehat{F}(y, t)=e^{\omega t} F(y, t), \\
\widehat{f}(y, t)=e^{\omega t} f(y, t), \quad \widehat{h}(y, t)=e^{\omega t} h(y, t) .
\end{array}
$$


The system satisfied by $(\widehat{u}, \widehat{p}, \widehat{F}, \widehat{f})$ is

$$
\begin{aligned}
& \frac{\partial \widehat{u}}{\partial t}-\omega \widehat{u}-\nu \Delta \widehat{u}+\nabla \widehat{p}=\widehat{\mathcal{F}}(\widehat{u}, \widehat{p}) \quad \text { and } \quad \operatorname{div} \widehat{u}=\widehat{\mathcal{G}}(\widehat{u})=\operatorname{div} \widehat{\mathbf{g}}(\widehat{u}) \quad \text { in } Q_{f}^{\infty}, \\
& \widehat{u}(0)=u_{0} \quad \text { in } \Omega_{f}, \quad \widehat{u}=0 \quad \text { on } \Sigma_{e}^{\infty}, \\
& \widehat{u}=\sum_{i=1}^{N_{c}} \widehat{f}_{i}(t) \xi_{i}(y) \quad \text { on } \Sigma_{s}^{\infty} \\
& \widehat{F}^{\prime}(t)=\omega \widehat{F}(t)+\widehat{f}(t), \quad \widehat{F}(0)=0, \\
& \widehat{f}^{\prime}(t)=\omega \widehat{f}(t)+\widehat{h}(t), \quad \widehat{f}(0)=0, \\
& \widehat{X}(y, t)=y+\int_{0}^{t} e^{-\omega s} \widehat{u}(y, s) \mathrm{d} s, \quad \text { for all } y \in \Omega_{f} \text { and all } t \in[0, \infty), \\
& \Omega_{f}(t)=\widehat{X}\left(\Omega_{f}, t\right), \\
& \widehat{Y}(\widehat{X}(y, t), t)=y, \quad y \in \Omega_{f} \quad \text { and } \quad \widehat{X}(\widehat{Y}(x, t), t)=x, \quad x \in \Omega_{f}(t), t \in[0, \infty) .
\end{aligned}
$$

where the nonlinear terms $\widehat{\mathcal{F}}, \widehat{\mathcal{G}}$, and $\widehat{\mathrm{g}}$ are defined by

$$
\begin{aligned}
& \widehat{\mathcal{F}}(\widehat{u}, \widehat{p})=e^{\omega t} \mathcal{F}_{1}\left(e^{-\omega t} \widehat{u}\right)+e^{\omega t} \mathcal{F}_{2}\left(e^{-\omega t} \widehat{u}\right)+e^{\omega t} \mathcal{F}_{3}\left(e^{-\omega t} \widehat{u}, e^{-\omega t} \widehat{p}\right), \\
& \widehat{\mathcal{G}}(\widehat{u})=e^{\omega t} \mathcal{G}\left(e^{-\omega t} \widehat{u}\right) \quad \text { and } \quad \widehat{\mathbf{g}}(\widehat{u})=e^{\omega t} \mathbf{g}\left(e^{-\omega t} \widehat{u}\right)
\end{aligned}
$$

We notice that $\widehat{X}(y, t)=X(y, t)$, and therefore $\widehat{Y}(x, t)=Y(x, t)$.

Let us notice that, if the functions $\left(\xi_{i}\right)_{1 \leq i \leq N_{c}}$ are chosen so that $\int_{\Gamma_{s}} \xi_{i} \cdot n \mathrm{~d} x=0$, for all $1 \leq i \leq N_{c}$, then

$$
\int_{\Gamma_{s}} \widehat{\mathbf{g}}(\widehat{u})(t) \cdot n \mathrm{~d} x=0
$$

Indeed, $\int_{\Gamma_{s}} \widehat{u}(t) \cdot n \mathrm{~d} x=\int_{\Gamma_{s}} \sum_{i=1}^{N_{c}} f_{i}(t) \xi_{i} \cdot n \mathrm{~d} x=0$ and

$$
\int_{\Gamma_{s}} \operatorname{det}\left(J_{\widehat{X}}\right) J_{\widehat{Y}} \widehat{u}(t) \cdot n \mathrm{~d} x=e^{\omega t} \int_{\Gamma_{s}(t)} u(t) \cdot \widetilde{n} \mathrm{~d} x=0
$$

The first equality in (1.9) is a consequence of Nanson's formula and the last one is a consequence of the fact that $\operatorname{div} u(t)=0$ in $\Omega_{f}(t)$.

In (1.7), $\widehat{h}=\left(\widehat{h}_{1}, \cdots, \widehat{h}_{N_{c}}\right)^{T}$ is the control, and the function $\xi=\left(\xi_{i}\right)_{1 \leq i \leq N_{c}}$ has to be suitably chosen in such a way that the linearized system associated with (1.7) is stabilizable by controls $h \in L^{2}\left(0, \infty ; \mathbb{R}^{N_{c}}\right)$. The equation $\widehat{F}^{\prime}=\omega \widehat{F}+\widehat{f}, \widehat{F}(0)=0$, is added to guarantee that $\left.\widehat{X}(\cdot, t)\right|_{\Gamma_{s}}$ tends to the identity with the exponential decay rate $\omega>0$, when $t$ tends to infinity. Thus the fluid domain $\Omega_{f}(t)$ tends to the initial configuration $\Omega_{f}$, with the exponential decay rate $\omega>0$, as $t$ tends to infinity. And the equation $\widehat{f}^{\prime}=\omega \widehat{f}+\widehat{h}, \widehat{f}(0)=0$, is added to improve the regularity of $\widehat{f}$. 
Let us notice that if $\widehat{h}$ is a control stabilizing system (1.7), then system (1.2) $)_{1-3}$ will be stabilized, with the exponential decay rate $\omega>0$, by the boundary deformation $X$ satisfying the differential equation

$$
\frac{\partial X}{\partial t}(y, t)=u(X(y, t), t), \quad X(y, 0)=y \quad \text { for all } y \in \Gamma_{s} .
$$

This is why we shall speak of stabilization by shape deformations of the fluid-obstacle interface.

Before stating the main result of the paper, we recall that, for $s \geq 0, H^{s}\left(\Omega_{f}\right)$ denotes the usual Sobolev spaces. We would like to underline that we shall make the abuse of notation by writing $H^{s}\left(\Omega_{f}\right)$ for $H^{s}\left(\Omega_{f} ; \mathbb{R}^{3}\right)$ or $H^{s}\left(\Omega_{f} ; \mathbb{R}^{3 \times 3}\right)$ for vector valued or matrix valued functions. The distinction between these three spaces should be clear from the context. We may also use the notation $H^{s}\left(\Omega_{f}\right)$ for $H^{s}\left(\Omega_{f} ; \mathbb{C}^{3}\right)$, for eigenvalue problems in which eigenvalues and eigenfunctions may be with complex values.

For $s>0$, the space $H^{s, s / 2}\left(Q_{f}^{\infty}\right)$ is nothing but $L^{2}\left(0, \infty ; H^{s}\left(\Omega_{f}\right)\right) \cap H^{s / 2}\left(0, \infty ; L^{2}\left(\Omega_{f}\right)\right)$. We shall also make the abuse of notation by writing $H^{s, s / 2}\left(Q_{f}^{\infty}\right)$ for $L^{2}\left(0, \infty ; H^{s}\left(\Omega_{f} ; \mathbb{R}^{3}\right)\right) \cap H^{s / 2}\left(0, \infty ; L^{2}\left(\Omega_{f} ; \mathbb{R}^{3}\right)\right)$.

Throughout the paper, we assume that

$$
\Omega_{f} \quad \text { is of class } C^{5} \text {. }
$$

The main result of the paper concerning the stabilization of the Navier-Stokes equations by boundary deformations is stated below.

Theorem 1.1. (Stabilization of the fluid equations in Lagrangian variables). Let $\omega>0$ be given fixed, and let $\left(\xi_{i}\right)_{1 \leq i \leq N_{c}}$ satisfy (3.2)-(3.3). Assume that $u_{0} \in H^{1+\ell}\left(\Omega_{f}\right) \cap H_{0}^{1}\left(\Omega_{f}\right)$ with $\ell \in\left(\frac{1}{2}, \frac{3}{2}\right)$, and $\operatorname{div} u_{0}=0$. If in addition $\left\|u_{0}\right\|_{H^{1+\ell}\left(\Omega_{f}\right)}$ is small enough, then there exists a control $h$ belonging to $H^{1+\ell / 2}\left(0, \infty ; \mathbb{R}^{N_{c}}\right)$, in feedback form, such that the system (1.7) admits a solution $(\widehat{u}, \widehat{F}, \widehat{f}, \widehat{X})$ belonging to

$$
H^{2+\ell, 1+\ell / 2}\left(Q_{f}^{\infty}\right) \times H^{3+\ell / 2}\left(0, \infty ; \mathbb{R}^{N_{c}}\right) \times H^{2+\ell / 2}\left(0, \infty ; \mathbb{R}^{N_{c}}\right) \times I_{\ell},
$$

where

$$
\begin{array}{r}
I_{\ell}=\left\{X \in L^{\infty}\left(0, \infty ; H^{2+\ell}\left(\Omega_{f} ; \mathbb{R}^{3}\right)\right) \cap H_{\mathrm{loc}}^{1}\left([0, \infty) ; H^{2+\ell}\left(\Omega_{f} ; \mathbb{R}^{3}\right)\right)\right. \\
\left.\mid \partial_{t} X \in L^{2}\left(0, \infty ; H^{2+\ell}\left(\Omega_{f}\right)\right) \cap H^{1}\left(0, \infty ; H^{\ell}\left(\Omega_{f}\right)\right)\right\} .
\end{array}
$$

The outline of the paper is as follows. We prove new regularity results for the Stokes system in Section 2. The stabilizability and the feedback stabilization of the linearized Navier-Stokes system controlled by boundary deformations is studied in Section 3. The associated closed-loop nonhomogeneous linear system is studied in Section 4. Section 5 is devoted to estimates of nonlinear terms of the Navier-Stokes system controlled by boundary deformations. Theorem 1.1 is proved in Section 6 by a fixed point method. In Section A, we have collected estimates on products of functions needed in Section 5.

As the existence of solution to the nonlinear closed loop system (1.7) is proved by the Banach fixed point Theorem, we only prove the uniqueness of the fixed point. This does not mean that the solution is unique. However, we think it is the case, and that the uniqueness can be proved as in [19] by showing that the system (6.1) admits a unique maximal solution. But due to the length of the proof in [19], we shall explore that issue in a forthcoming paper.

\section{The Stokes equations}

In this section, we are going to state results very similar to those in [25], except that in [25] all the regularity results are stated for equations defined over a bounded time interval $(0, T)$, while, here, we are going to prove results for equations defined over the time interval $(0, \infty)$. 


\subsection{Some function spaces}

We introduce the following function spaces

$$
\begin{aligned}
& V_{n}^{0}\left(\Omega_{f}\right)=\left\{u \in L^{2}\left(\Omega_{f} ; \mathbb{R}^{3}\right) \mid \operatorname{div} u=0 \text { in } \Omega_{f}, u \cdot n=0 \text { on } \partial \Omega_{f}\right\}, \\
& V^{0}\left(\Omega_{f}\right)=\left\{u \in L^{2}\left(\Omega_{f} ; \mathbb{R}^{3}\right) \mid \operatorname{div} u=0 \text { in } \Omega_{f}\right\}, \\
& V_{0}^{1}\left(\Omega_{f}\right)=H_{0}^{1}\left(\Omega_{f} ; \mathbb{R}^{3}\right) \cap V_{n}^{0}\left(\Omega_{f}\right), \\
& V^{2}\left(\Omega_{f}\right)=\left\{u \in H^{2}\left(\Omega_{f} ; \mathbb{R}^{3}\right) \mid \operatorname{div} u=0 \text { in } \Omega_{f}\right\} .
\end{aligned}
$$

We also introduce the dual spaces of $V_{0}^{1}\left(\Omega_{f}\right)$ and of $V^{2}\left(\Omega_{f}\right) \cap V_{0}^{1}\left(\Omega_{f}\right)$ with $V_{n}^{0}\left(\Omega_{f}\right)$ as pivot space:

$$
\begin{aligned}
& V_{0}^{1}\left(\Omega_{f}\right) \hookrightarrow V_{n}^{0}\left(\Omega_{f}\right) \hookrightarrow V^{-1}\left(\Omega_{f}\right)=\left(V_{0}^{1}\left(\Omega_{f}\right)\right)^{\prime} \\
& V^{2}\left(\Omega_{f}\right) \cap V_{0}^{1}\left(\Omega_{f}\right) \hookrightarrow V_{n}^{0}\left(\Omega_{f}\right) \hookrightarrow V^{-2}\left(\Omega_{f}\right)=\left(V^{2}\left(\Omega_{f}\right) \cap V_{0}^{1}\left(\Omega_{f}\right)\right)^{\prime} .
\end{aligned}
$$

\subsection{The Leray projector}

Let us recall that

$$
L^{2}\left(\Omega_{f} ; \mathbb{R}^{3}\right)=V_{n}^{0}\left(\Omega_{f}\right) \oplus \nabla H^{1}\left(\Omega_{f}\right)
$$

where $\nabla H^{1}\left(\Omega_{f}\right)$ is the subspace of functions belonging to $L^{2}\left(\Omega_{f} ; \mathbb{R}^{3}\right)$ and which are the gradients of functions belonging to $H^{1}\left(\Omega_{f}\right)$. The Leray projector $P$ is the orthogonal projector from $L^{2}\left(\Omega_{f} ; \mathbb{R}^{3}\right)$ to $V_{n}^{0}\left(\Omega_{f}\right)$. Let us recall that, for all $u \in L^{2}\left(\Omega_{f} ; \mathbb{R}^{3}\right), P u=u-\nabla(\zeta+\pi)$, where $\zeta$ is the solution to the elliptic equation

$$
\zeta \in H^{1}\left(\Omega_{f}\right), \quad \Delta \zeta=\operatorname{div} u \quad \text { in } \Omega_{f}, \quad \zeta=0 \quad \text { on } \partial \Omega_{f},
$$

and $\pi$ satisfies

$$
\pi \in H^{1}\left(\Omega_{f}\right), \quad \Delta \pi=0 \quad \text { in } \Omega_{f}, \quad \frac{\partial \pi}{\partial n}=(u-\nabla \zeta) \cdot n \quad \text { on } \partial \Omega_{f} .
$$

As usual $(u-\nabla \zeta) \cdot n$ is well defined in $H^{-1 / 2}(\Gamma)$, the dual of $H^{1 / 2}(\Gamma)$, since $(u-\nabla \zeta) \in L^{2}\left(\Omega_{f} ; \mathbb{R}^{3}\right)$ and $\operatorname{div}(u-\nabla \zeta)=0$ in $\Omega_{f}$.

The operator $P$ may be continuously extended as an operator belonging to $\mathcal{L}\left(H^{-1}\left(\Omega_{f} ; \mathbb{R}^{3}\right), V^{-1}\left(\Omega_{f}\right)\right)$, still denoted by $P$ for simplicity, defined by

$$
\langle P v, \phi\rangle_{V^{-1}\left(\Omega_{f}\right), V_{0}^{1}\left(\Omega_{f}\right)}=\langle v, \phi\rangle_{H^{-1}\left(\Omega_{f} ; \mathbb{R}^{3}\right), H_{0}^{1}\left(\Omega_{f} ; \mathbb{R}^{3}\right)},
$$

for all $v \in H^{-1}\left(\Omega_{f} ; \mathbb{R}^{3}\right)$ and all $\phi \in V_{0}^{1}\left(\Omega_{f}\right)$. Similarly $P$ may also be continuously extended as an operator belonging to $\mathcal{L}\left(\left(H^{2} \cap H_{0}^{1}\left(\Omega_{f} ; \mathbb{R}^{3}\right)\right)^{\prime}, V^{-2}\left(\Omega_{f}\right)\right)$, still denoted by $P$ for simplicity, defined by

$$
\langle P v, \phi\rangle_{V^{-2}\left(\Omega_{f}\right), V_{0}^{1}\left(\Omega_{f}\right) \cap V^{2}\left(\Omega_{f}\right)}=\langle v, \phi\rangle_{\left(\left(H^{2} \cap H_{0}^{1}\right)\left(\Omega_{f} ; \mathbb{R}^{3}\right)\right)^{\prime},\left(H^{2} \cap H_{0}^{1}\right)\left(\Omega_{f} ; \mathbb{R}^{3}\right)},
$$

for all $v \in\left(H^{2} \cap H_{0}^{1}\left(\Omega_{f} ; \mathbb{R}^{3}\right)\right)^{\prime}$ and all $\phi \in V_{0}^{1}\left(\Omega_{f}\right) \cap V^{2}\left(\Omega_{f}\right)$.

The Stokes operator is the unbounded operator $(A, D(A))$ in $V_{n}^{0}\left(\Omega_{f}\right)$, defined by $D(A)=V_{0}^{1}\left(\Omega_{f}\right) \cap V^{2}\left(\Omega_{f}\right)$ and $A=\nu P \Delta$. With the extrapolation method, the Stokes operator may be extended to an unbounded operator in $V^{-1}\left(\Omega_{f}\right)$ and in $V^{-2}\left(\Omega_{f}\right)$ as follows. The operator $\left(A, D\left(A ; V^{-1}\left(\Omega_{f}\right)\right)\right)$ is defined by $D\left(A ; V^{-1}\left(\Omega_{f}\right)\right)=$ 
$V_{0}^{1}\left(\Omega_{f}\right)$ and

$$
\langle A v, \phi\rangle_{V^{-1}\left(\Omega_{f}\right), V_{0}^{1}\left(\Omega_{f}\right)}=-\nu \int_{\Omega_{f}} \nabla v: \nabla \phi \mathrm{d} x
$$

for all $v \in V_{0}^{1}\left(\Omega_{f}\right)$ and all $\phi \in V_{0}^{1}\left(\Omega_{f}\right)$.

The operator $\left(A, D\left(A ; V^{-2}\left(\Omega_{f}\right)\right)\right.$ is defined by $D\left(A ; V^{-2}\left(\Omega_{f}\right)\right)=V_{n}^{0}\left(\Omega_{f}\right)$ and

$$
\langle A v, \phi\rangle_{V^{-2}\left(\Omega_{f}\right), V_{0}^{1}\left(\Omega_{f}\right) \cap V^{2}\left(\Omega_{f}\right)}=-\nu \int_{\Omega_{f}} v \cdot \Delta \phi \mathrm{d} x
$$

for all $v \in V_{n}^{0}\left(\Omega_{f}\right)$ and all $\phi \in V_{0}^{1}\left(\Omega_{f}\right) \cap V^{2}\left(\Omega_{f}\right)$.

Remark 2.1. Let us notice that, since $\Omega_{f}$ is of class $C^{5}$, the Leray projector $P$ is continuous from $H^{2+\ell}\left(\Omega_{f} ; \mathbb{R}^{3}\right)$ into $V_{n}^{0}\left(\Omega_{f}\right) \cap H^{2+\ell}\left(\Omega_{f} ; \mathbb{R}^{3}\right)$ for all $\ell \in\left(\frac{1}{2}, \frac{3}{2}\right)$. Indeed, if $u$ belongs to $H^{4}\left(\Omega_{f} ; \mathbb{R}^{3}\right)$ (resp. $H^{2}\left(\Omega_{f} ; \mathbb{R}^{3}\right)$ ), due to elliptic regularity results and the fact that $\Omega_{f}$ is of class $C^{5}$ (see, e.g., [31], Thm. 2.24), the solution $\zeta$ to (2.2) and the solution $\pi$ to (2.3) belong to $H^{5}\left(\Omega_{f}\right)$ (resp. $H^{3}\left(\Omega_{f} ; \mathbb{R}^{3}\right)$ ) (the nonhomogeneous boundary condition for the solution $\pi$ to (2.3) can be treated by a lifting). Since $3<3+\ell<5$, if $u$ belongs to $H^{2+\ell}\left(\Omega_{f} ; \mathbb{R}^{3}\right)$, by interpolation, the solution $\zeta$ to $(2.2)$ and the solution $\pi$ to (2.3) belong to $H^{3+\ell}\left(\Omega_{f}\right)$. Thus $P u=u-\nabla(\zeta+\pi)$ belongs to $V_{n}^{0}\left(\Omega_{f}\right) \cap H^{2+\ell}\left(\Omega_{f} ; \mathbb{R}^{3}\right)$.

Assumption (1.11) on the regularity of $\Omega_{f}$ is also used in (3.3), see Remark 3.1.

\subsection{Regularity results for the Stokes equations}

We study the following Stokes system with Dirichlet boundary conditions and a nonhomogeneous divergence condition

$$
\begin{aligned}
& \frac{\partial v}{\partial t}-\nu \Delta v+\nabla q=\mathcal{F} \quad \text { in } Q_{f}^{\infty}, \\
& \operatorname{div} v=g=\operatorname{div} \mathbf{g} \quad \text { in } Q_{f}^{\infty}, \\
& v(0)=v_{0} \text { in } \Omega_{f}, \quad v=0 \quad \text { on } \Sigma_{e}^{\infty}, \\
& v=\xi \quad \text { on } \Sigma_{s}^{\infty},
\end{aligned}
$$

with the compatibility condition

$$
\int_{\Gamma_{s}} \boldsymbol{\xi}(t) \cdot n \mathrm{~d} x=\int_{\Omega} g(t) \mathrm{d} x=\int_{\Gamma_{s}} \mathbf{g}(t) \cdot n \mathrm{~d} x \quad \text { for all } t \in[0, \infty) .
$$

We recall that $\ell \in\left(\frac{1}{2}, \frac{3}{2}\right)$. We introduce the spaces

$$
\begin{aligned}
& E_{\ell}=\left\{u \in H^{2+\ell, 1+\ell / 2}\left(Q_{f}^{\infty}\right)|u|_{\Sigma_{s}^{\infty}} \in H^{1+\ell / 2}\left(0, \infty ; H^{3 / 2+\ell}\left(\Gamma_{s}\right)\right)\right\}, \\
& F_{\ell}=H^{\ell, \ell / 2}\left(Q_{f}^{\infty}\right), \\
& G_{\ell}=L^{2}\left(0, \infty ; H^{1+\ell}\left(\Omega_{f}\right)\right) \cap H^{\ell / 2}\left(0, \infty ; H^{1}\left(\Omega_{f}\right)\right), \\
& \mathbf{G}_{\ell}=H^{1+\ell / 2}\left(0, \infty ; L^{2}\left(\Omega_{f}\right)\right), \\
& P_{\ell}=\left\{p \in L^{2}\left(Q_{f}^{\infty}\right) \mid \nabla p \in H^{\ell, \ell / 2}\left(Q_{f}^{\infty}\right), \quad \int_{\Omega_{f}} p(x, t) \mathrm{d} x=0 \text { for all } t \in(0, \infty)\right\} .
\end{aligned}
$$


The norm in $E_{\ell}$ will be denoted by $\|\cdot\|_{E_{\ell}}$, and it is defined by

$$
u \longmapsto\|u\|_{E_{\ell}}=\|u\|_{H^{2+\ell, 1+\ell / 2}\left(Q_{f}^{\infty}\right)}+\left\|\left.u\right|_{\Sigma_{s}^{\infty}}\right\|_{H^{1+\ell / 2}\left(0, \infty ; H^{3 / 2+\ell}\left(\Gamma_{s}\right)\right)} .
$$

The usual norms in $F_{\ell}, G_{\ell}$, and $\mathbf{G}_{\ell}$ will be respectively denoted by $\|\cdot\|_{F_{\ell}},\|\cdot\|_{G_{\ell}}$, and $\|\cdot\|_{\mathbf{G}_{\ell}}$. The norm in $P_{\ell}$ is $p \mapsto\|\nabla p\|_{F_{\ell}}$.

Remark 2.2. Let us notice that if $u$ belongs to $H^{2+\ell, 1+\ell / 2}\left(Q_{f}^{\infty}\right),\left.u\right|_{\Sigma_{s}^{\infty}}$, the trace of $u$ on $\Sigma_{s}^{\infty}$, belongs to $H^{3 / 2+\ell, 3 / 4+\ell / 2}\left(\Sigma_{s}^{\infty}\right)=L^{2}\left(0, \infty ; H^{3 / 2+\ell}\left(\Gamma_{s}\right)\right) \cap H^{3 / 4+\ell / 2}\left(0, \infty ; L^{2}\left(\Gamma_{s}\right)\right)$. Since we need to consider velocity fields $u$ whose trace on $\Sigma_{s}^{\infty}$ belongs to $H^{1+\ell / 2}\left(0, \infty ; H^{3 / 2+\ell}\left(\Gamma_{s}\right)\right)$, this additional condition has to be included in the definition of $E_{\ell}$.

We want to prove the following theorem.

Theorem 2.3. If $\ell \in\left(\frac{1}{2}, \frac{3}{2}\right)$,

$$
\begin{aligned}
& \mathcal{F} \in H^{\ell, \ell / 2}\left(Q_{f}^{\infty}\right), \quad v_{0} \in H^{1+\ell}\left(\Omega_{f}\right) \cap H_{0}^{1}\left(\Omega_{f}\right), \quad \operatorname{div} v_{0}=0, \\
& \boldsymbol{\xi} \in H^{1+\ell / 2}\left(0, \infty ; H^{3 / 2+\ell}\left(\Gamma_{s}\right)\right), \\
& \mathbf{g} \in H^{1+\ell / 2}\left(0, \infty ; L^{2}\left(\Omega_{f}\right)\right), \quad \mathbf{g} \in C_{b}\left([0, \infty) ; H^{1+\ell}\left(\Omega_{f}\right)\right),\left.\quad \mathbf{g}\right|_{\Sigma_{e}^{\infty}}=0, \\
& g \in L^{2}\left(0, \infty ; H^{1+\ell}\left(\Omega_{f}\right)\right) \cap H^{\ell / 2}\left(0, \infty ; H^{1}\left(\Omega_{f}\right)\right),
\end{aligned}
$$

and if in addition the following compatibility conditions are satisfied

$$
\begin{aligned}
& \mathcal{F}(\cdot, 0)=0 \quad \text { in } \Omega_{f}, \\
& \mathbf{g}(\cdot, 0)=0 \quad \text { in } \Omega_{f}, \quad \boldsymbol{\xi}(\cdot, 0)=0 \quad \text { on } \Gamma_{s}, \\
& \text { and } \quad \int_{\Omega} g \mathrm{~d} x=\int_{\Gamma_{s}} \mathbf{g} \cdot n \mathrm{~d} x=\int_{\Gamma_{s}} \boldsymbol{\xi} \cdot n \mathrm{~d} x,
\end{aligned}
$$

then the solution to system (2.4) satisfies

$$
\begin{aligned}
& \|v\|_{E_{\ell}}+\|\nabla q\|_{F_{\ell}} \\
& \leq C\left(\|\mathcal{F}\|_{F_{\ell}}+\|g\|_{G_{\ell}}+\|\mathbf{g}\|_{\mathbf{G}_{\ell}}+\|\boldsymbol{\xi}\|_{H^{1+\ell / 2}\left(0, \infty ; H^{3 / 2+\ell}\left(\Gamma_{s}\right)\right)}+\left\|v_{0}\right\|_{H^{1+\ell}\left(\Omega_{f}\right)}\right) .
\end{aligned}
$$

Remark 2.4. A similar theorem is proved in Theorem 3.2 of [1] in the case where $1<\ell<3 / 2$, and $0<T<\infty$. Another version of Theorem 2.3 is stated in Theorem 1.1 of [27], still in the case where $0<T<\infty$.

To prove Theorem 2.3, we split the solution $(v, q)$ of equation $(2.4)$ in the form $(v, q)=(\widetilde{v}, \widetilde{q})+(\widehat{v}, \widehat{q})$, where $(\widetilde{v}, \widetilde{q})$ is solution of

$$
\begin{aligned}
& \frac{\partial \widetilde{v}}{\partial t}-\nu \Delta \widetilde{v}+\nabla \widetilde{q}=0 \quad \text { in } Q_{f}^{\infty}, \\
& \operatorname{div} \widetilde{v}=g=\operatorname{div} \mathbf{g} \text { in } Q_{f}^{\infty}, \\
& \widetilde{v}(0)=0 \text { in } \Omega_{f}, \quad \widetilde{v}=0 \quad \text { on } \Sigma_{e}^{\infty}, \\
& \widetilde{v}=\xi \quad \text { on } \Sigma_{s}^{\infty},
\end{aligned}
$$


and $(\widehat{v}, \widehat{q})$ is solution of

$$
\begin{aligned}
& \frac{\partial \widehat{v}}{\partial t}-\nu \Delta \widehat{v}+\nabla \widehat{q}=\mathcal{F} \quad \text { in } Q_{f}^{\infty}, \\
& \operatorname{div} \widehat{v}=0 \quad \text { in } Q_{f}^{\infty}, \\
& \widehat{v}(0)=v_{0} \text { in } \Omega_{f}, \quad \widehat{v}=0 \quad \text { on } \Sigma_{e}^{\infty}, \\
& \widehat{v}=0 \quad \text { on } \Sigma_{s}^{\infty} .
\end{aligned}
$$

\subsection{Equation (2.8)}

Proposition 2.5. If $\mathrm{g}$, $\mathbf{g}$, and $\boldsymbol{\xi}$ obey the assumptions of Theorem 2.3, then the solution to system (2.8) satisfies $\widetilde{v} \in E_{\ell}, \nabla \widetilde{q} \in F_{\ell}$ and

$$
\begin{aligned}
& \|\widetilde{v}\|_{E_{\ell}}+\|\nabla \widetilde{q}\|_{F_{\ell}} \\
& \leq C\left(\|\mathbf{g}\|_{H^{1+\ell / 2}\left(0, \infty ; L^{2}\left(\Omega_{f}\right)\right)}+\|g\|_{H^{\ell / 2}\left(0, \infty ; H^{1}\left(\Omega_{f}\right)\right)}+\|g\|_{L^{2}\left(0, \infty ; H^{1+\ell / 2}\left(\Omega_{f}\right)\right)}+\|\boldsymbol{\xi}\|_{H^{1+\ell / 2}\left(0, \infty ; H^{3 / 2+\ell}\left(\Gamma_{s}\right)\right)} .\right.
\end{aligned}
$$

Proof. For $g=\operatorname{div} \mathbf{g}$ and $\boldsymbol{\xi}$ satisfying the compatibility condition $(2.6)$, we define $L(g, \boldsymbol{\xi})$ and $L_{p}(g, \boldsymbol{\xi})$ by

$$
\left(L, L_{p}\right) \in \mathcal{L}\left(L^{2}\left(\Omega_{f}\right) \times L^{2}\left(\Gamma_{s}\right), L^{2}\left(\Omega_{f} ; \mathbb{R}^{3}\right) \times L^{2}\left(\Omega_{f}\right)\right) \text { and } L(g, \boldsymbol{\xi})=u, \text { and } L_{p}(g, \boldsymbol{\xi})=\rho,
$$

where $(u, \rho)$ is the solution to the following stationary problem

$$
\begin{aligned}
& -\nu \Delta u+\nabla \rho=0 \quad \text { in } \Omega_{f}, \\
& \operatorname{div}(u)=g=\operatorname{div} \mathbf{g} \quad \text { in } \Omega_{f}, \\
& u=0 \quad \text { on } \Gamma_{e}, \quad u=\boldsymbol{\xi} \text { on } \Gamma_{s} .
\end{aligned}
$$

When $\boldsymbol{\xi} \in H^{1+\ell / 2}\left(0, \infty ; H^{3 / 2+\ell}\left(\Gamma_{s}\right)\right)$ and $\mathbf{g} \in \mathbf{G}_{\ell}$, since $\boldsymbol{\xi}$ and $\mathbf{g}$ depend on the time variable $t$, we shall also emphasize the dependence of $u$ and $\rho$ with respect to $t$ by setting $L(g(t), \boldsymbol{\xi}(t))=u(t)$ and $L_{p}(g(t), \boldsymbol{\xi}(t))=\rho(t)$.

From Corollary 8.4 of [23], it follows that $u(t)=L(g(t), \boldsymbol{\xi}(t))$ satisfies

$$
\|u(t)\|_{L^{2}\left(\Omega_{f}\right)} \leq C\left(\|\mathbf{g}(t)\|_{L^{2}\left(\Omega_{f}\right)}+\|\boldsymbol{\xi}(t)\|_{L^{2}\left(\Gamma_{s}\right)}\right) .
$$

Thus, we obtain

$$
\|u\|_{H^{1+\ell / 2}\left(0, \infty ; L^{2}\left(\Omega_{f}\right)\right)} \leq C\left(\|\mathbf{g}\|_{H^{1+\ell / 2}\left(0, \infty ; L^{2}\left(\Omega_{f}\right)\right)}+\|\boldsymbol{\xi}\|_{H^{1+\ell / 2}\left(0, \infty ; L^{2}\left(\Gamma_{s}\right)\right)}\right) .
$$

We also have (see [8])

$$
\|u\|_{H^{\ell / 2}\left(0, \infty ; H^{2}\left(\Omega_{f}\right)\right)}+\|\nabla \rho\|_{H^{\ell / 2}\left(0, \infty ; L^{2}\left(\Omega_{f}\right)\right)} \leq C\left(\|g\|_{H^{\ell / 2}\left(0, \infty ; H^{1}\left(\Omega_{f}\right)\right)}+\|\boldsymbol{\xi}\|_{H^{1+\ell / 2}\left(0, \infty ; H^{3 / 2}+\ell\left(\Gamma_{s}\right)\right)}\right)
$$

and

$$
\|u\|_{L^{2}\left(0, \infty ; H^{2+\ell}\left(\Omega_{f}\right)\right)}+\|\nabla \rho\|_{L^{2}\left(0, \infty ; H^{\ell}\left(\Omega_{f}\right)\right)} \leq C\left(\|g\|_{L^{2}\left(0, \infty ; H^{1+\ell / 2}\left(\Omega_{f}\right)\right)}+\|\boldsymbol{\xi}\|_{H^{1+\ell / 2}\left(0, \infty ; H^{3 / 2+\ell}\left(\Gamma_{s}\right)\right)}\right) .
$$

Therefore the solutions $u$ to $(2.11)$ belongs to $E_{\ell}$, and $\nabla \rho \in F_{\ell}$. 
We look for the solution $(\widetilde{v}, \widetilde{q})$ to $(2.8)$ in the form $(\widetilde{v}, \widetilde{q})=(u, \rho)+(\widetilde{w}, \widetilde{\rho})$, where $(u(t), \rho(t))=$ $\left(L(g(t), \boldsymbol{\xi}(t)), L_{p}(g(t), \boldsymbol{\xi}(t))\right)$. Thus $(\widetilde{w}, \widetilde{\rho})$ is solution of

$$
\begin{aligned}
& \frac{\partial \widetilde{w}}{\partial t}-\nu \Delta \widetilde{w}+\nabla \widetilde{\rho}=-\frac{\partial u}{\partial t} \quad \text { in } Q_{f}^{\infty}, \\
& \operatorname{div} \widetilde{w}=0 \quad \text { in } Q_{f}^{\infty}, \\
& \widetilde{w}(0)=0 \text { in } \Omega_{f}, \quad \widetilde{w}=0 \quad \text { on } \Sigma_{e}^{\infty}, \\
& \widetilde{w}=0 \quad \text { on } \Sigma_{s}^{\infty} .
\end{aligned}
$$

Since $u$ belongs to $E_{\ell}, \frac{\partial u}{\partial t}$ belongs to $F_{\ell}$, and, due to Theorem 5.3 of [8] (see also the arguments in the proof of Prop. 2.6), we have the estimate

$$
\|\widetilde{w}\|_{E_{\ell}}+\|\nabla \widetilde{\rho}\|_{F_{\ell}} \leq C\left\|\frac{\partial u}{\partial t}\right\|_{F_{\ell}}
$$

From the above estimates for $(u, \rho)$ and $(\widetilde{w}, \widetilde{\rho})$, it follows that

$$
\begin{aligned}
\|\widetilde{v}\|_{E_{\ell}}+\|\nabla \widetilde{q}\|_{F_{\ell}} \leq & C\left(\|\mathbf{g}\|_{H^{1+\ell / 2}\left(0, \infty ; L^{2}\left(\Omega_{f}\right)\right)}+\|g\|_{H^{\ell / 2}\left(0, \infty ; H^{1}\left(\Omega_{f}\right)\right)}\right. \\
& +\|g\|_{L^{2}\left(0, \infty ; H^{1+\ell}\left(\Omega_{f}\right)\right)}+\|\boldsymbol{\xi}\|_{H^{1+\ell / 2}\left(0, \infty ; H^{3 / 2+\ell}\left(\Gamma_{s}\right)\right)}
\end{aligned}
$$

\subsection{Equation (2.9)}

Proposition 2.6. If $\mathcal{F}$ and $u_{0}$ obey the assumptions of Theorem 2.3, then the solution to system (2.9) satisfies $\widehat{v} \in E_{\ell}, \nabla \widehat{q} \in F_{\ell}$ and

$$
\|\widehat{v}\|_{E_{\ell}}+\|\nabla \widehat{q}\|_{F_{\ell}} \leq C\left(\left\|v_{0}\right\|_{H^{1+\ell}\left(\Omega_{f}\right)}+\|\mathcal{F}\|_{F_{\ell}}\right) .
$$

Proof. This is a classical regularity result for the Stokes equation. However, since we need estimates over the time interval $(0, \infty)$ we give the main arguments of the proof. (See [8] for estimates over a bounded time interval.) Let us denote by $v_{v_{0}, \mathcal{F}}$ the solution to (2.9), and let us set $v_{v_{0}}=v_{v_{0}, 0}$ and $v_{\mathcal{F}}=v_{0, \mathcal{F}}$. The estimate of $v_{v_{0}}$ in $E_{\ell}$ follows from the fact that the condition $v_{0} \in H^{1+\ell}\left(\Omega_{f}\right) \cap V_{0}^{1}\left(\Omega_{f}\right)$ is equivalent to $v_{0} \in D\left((-A)^{\ell / 2+1 / 2}\right)$. Thus we can apply ([2], Thm. 3.1, II-1) combined with Theorem 2.2, II-3 of [2] and the isomorphism result stated in ([2], (2.19), II-3).

To study the regularity of $v_{\mathcal{F}}$, we consider the case where $\mathcal{F} \in H^{1}\left(0, \infty ; L^{2}\left(\Omega_{f} ; \mathbb{R}^{3}\right)\right)$, and we are going to prove that

$$
\begin{aligned}
& \left\|v_{\mathcal{F}}\right\|_{H^{1}\left(0, \infty ; V_{n}^{0}\left(\Omega_{f}\right)\right)} \leq C\|\mathcal{F}\|_{L^{2}\left(0, \infty ; L^{2}\left(\Omega_{f} ; \mathbb{R}^{3}\right)\right)}, \\
& \text { and } \\
& \left\|v_{\mathcal{F}}\right\|_{H^{2}\left(0, \infty ; V_{n}^{0}\left(\Omega_{f}\right)\right)} \leq C\|\mathcal{F}\|_{H^{1}\left(0, \infty ; L^{2}\left(\Omega_{f} ; \mathbb{R}^{3}\right)\right)} .
\end{aligned}
$$

If (2.15) is proved, by interpolation it follows that

$$
\left\|v_{\mathcal{F}}\right\|_{H^{1+\ell / 2}\left(0, \infty ; V_{n}^{0}\left(\Omega_{f}\right)\right)} \leq C\|\mathcal{F}\|_{H^{\ell / 2}\left(0, \infty ; L^{2}\left(\Omega_{f} ; \mathbb{R}^{3}\right)\right)}
$$


By applying Theorem 3.1, II-1 of [2] combined with Theorem 2.2, II-3 of [2], we first obtain (2.15) 1 . To prove $(2.15)_{2}$, using that $\mathcal{F}(0)=0$ and $v_{\mathcal{F}}(0)=0$, we have

$$
\frac{\mathrm{d}}{\mathrm{d} t} \widehat{v}_{\mathcal{F}}+A \widehat{v}_{\mathcal{F}}=P \widehat{\mathcal{F}}, \quad \text { over }(-1, \infty), \quad \widehat{v}_{\mathcal{F}}(-1)=0
$$

where $\widehat{v}_{\mathcal{F}}$ is the extension of $v_{\mathcal{F}}$ by zero to $(-1,0)$, and $\widehat{\mathcal{F}}$ is the extension of $\mathcal{F}$ by zero to $(-1,0)$. Thus the equation for $w=\frac{\mathrm{d}}{\mathrm{d} t} \widehat{v}_{\mathcal{F}}$ is

$$
\frac{\mathrm{d}}{\mathrm{d} t} w+A w=P \frac{\mathrm{d}}{\mathrm{d} t} \widehat{\mathcal{F}}, \quad \text { over }(-1, \infty), \quad w(-1)=0 .
$$

By applying Theorem 3.1, II-1 of [2] combined with Theorem 2.2, II-3 of [2], we obtain

$$
\|w\|_{H^{1}\left(-1, \infty ; V_{n}^{0}\left(\Omega_{f}\right)\right)} \leq C\left\|\frac{\mathrm{d}}{\mathrm{d} t} \mathcal{F}\right\|_{L^{2}\left(0, \infty ; L^{2}\left(\Omega_{f} ; \mathbb{R}^{3}\right)\right)},
$$

from which we deduce $(2.15)_{2}$. With $(2.16)$ and the equation $A v_{\mathcal{F}}=P \mathcal{F}-\partial_{t} v_{\mathcal{F}}$, we deduce that

$$
\left\|v_{\mathcal{F}}\right\|_{H^{\ell / 2}\left(0, \infty ; V^{2}\left(\Omega_{f}\right) \cap V_{0}^{1}\left(\Omega_{f}\right)\right)} \leq C\|\mathcal{F}\|_{F_{\ell}} .
$$

By interpolation, with (2.16) and (2.17), we obtain

$$
\left\|v_{\mathcal{F}}\right\|_{H^{1}\left(0, \infty ; H^{\ell}\left(\Omega_{f}\right)\right)} \leq C\|\mathcal{F}\|_{F_{\ell}} .
$$

Next using $A v_{\mathcal{F}}=P \mathcal{F}-\partial_{t} v_{\mathcal{F}}$, we deduce an estimate of $v_{\mathcal{F}}$ in $L^{2}\left(0, \infty ; V^{2+\ell}\left(\Omega_{f}\right)\right)$. The estimate for the pressure is derived from elliptic regularity results for the velocity and the pressure in the Stokes equation. The proof is complete.

End of proof of Theorem 2.3. The estimates of Theorem 2.3 clearly follow from Propositions 2.5 and 2.6.

\section{Stabilizability of The EXTEnded System}

The eigenfunctions of $A+\omega I$ corresponding to the eigenvalue $\lambda$ are the solutions $(\phi, \psi, \lambda) \in D(A) \times$ $H^{1}\left(\Omega_{f}\right) / \mathbb{R} \times \mathbb{R}$ of the system

$$
\begin{aligned}
& (\phi, \nabla \psi) \neq(0,0), \\
& \lambda \phi-\operatorname{div} \sigma(\phi, \psi)-\omega \phi=0, \\
& \operatorname{div} \phi=0 \quad \text { in } \Omega_{f}, \\
& \phi=0 \text { on } \Gamma_{s}, \quad \phi=0 \text { on } \Gamma_{e} .
\end{aligned}
$$

The triplet $(\phi, \psi, \lambda) \in D(A) \times H^{1}\left(\Omega_{f}\right) / \mathbb{R} \times \mathbb{R}$ is a solution to system $(3.1)$ if and only if $(\phi, \lambda-\omega) \in D(A) \times \mathbb{R}$ is a pair of eigenfunction and eigenvalue of the Stokes operator $(A, D(A))$. Thus, for $\omega>0$ given fixed, if $(\phi, \psi, \lambda) \in D(A) \times H^{1}\left(\Omega_{f}\right) / \mathbb{R} \times \mathbb{R}$ is a solution to system (3.1), then $\lambda-\omega<0$.

We choose the family $\left(\xi_{i}\right)_{1 \leq i \leq N_{c}}$ so that

$$
\int_{\Gamma_{s}} \xi_{i} \cdot n \mathrm{~d} x=0 \quad \text { for all } 1 \leq i \leq N_{c}
$$


and

For $1 \leq i \leq N_{c}$, the function $\xi_{i}$ belongs to $H^{3 / 2+\ell}\left(\Gamma_{s}\right)$ and

$$
\begin{aligned}
& \operatorname{span}\left\{\xi_{1}, \cdots, \xi_{N_{c}}\right\} \\
& \supset \operatorname{span}\left\{\left.\sigma(\phi, \psi) n\right|_{\Gamma_{s}}-\frac{n}{\left|\Gamma_{s}\right|} \int_{\Gamma_{s}} \sigma(\phi, \psi) n \cdot n \mathrm{~d} x \mid(\lambda, \phi, \psi) \text { is solution of (3.1) with } \lambda \geq 0\right\} .
\end{aligned}
$$

Remark 3.1. The space $\operatorname{span}\{(\phi, \psi) \mid(\lambda, \phi, \psi)$ is solution of (3.1) with $\lambda \geq 0\}$ is of finite dimension. Since $\Omega_{f}$ is of class $C^{5}$, if $(\lambda, \phi, \psi)$ is solution of (3.1) with $\lambda \geq 0$, then $(\phi, \psi) \in H^{5}\left(\Omega_{f} ; \mathbb{R}^{3}\right) \times H^{4}\left(\Omega_{f}\right)$ and $x \mapsto n(x)$ is of class $C^{4}$ on $\Gamma_{s}$. Thus $x \longmapsto \zeta(x)=[\sigma(\phi, \psi) n](x)-\frac{n(x)}{\left|\Gamma_{s}\right|} \int_{\Gamma_{s}} \sigma(\phi, \psi) n \cdot n \mathrm{~d} s$ belongs to $H^{7 / 2}\left(\Gamma_{s} ; \mathbb{R}^{3}\right)$, and satisfies $\int_{\Gamma_{s}} \zeta \cdot n \mathrm{~d} x=0$. Since $3 / 2+\ell<7 / 2$, we can always choose the family $\left\{\xi_{1}, \cdots, \xi_{N_{c}}\right\}$ in (3.3) so that the functions $\left(\xi_{i}\right)_{1 \leq i \leq N_{c}}$ belong to $H^{3 / 2+\ell}\left(\Gamma_{s} ; \mathbb{R}^{3}\right)$ and satisfy $(3.2)$.

\subsection{The extended system}

Let $\left\{\xi_{1}, \cdots, \xi_{N_{c}}\right\}$ be a family satisfying (3.3), and consider the extended system

$$
\begin{aligned}
& v_{t}-\operatorname{div} \sigma(v, p)-\omega v=0, \\
& \operatorname{div} v=0 \quad \text { in } Q_{f}^{\infty}, \\
& v=\sum_{i=1}^{N_{c}} f_{i}(t) \xi_{i}(x) \text { on } \Sigma_{s}^{\infty}, \quad v=0 \quad \text { on } \Sigma_{0}^{\infty}, \quad v(0)=v_{0} \text { in } \Omega_{f}, \\
& F^{\prime}=\omega F+f, \quad F(0)=0, \\
& f^{\prime}=\omega f+h, \quad f(0)=0 .
\end{aligned}
$$

We introduce the unbounded operator $\left(\mathcal{A}_{\omega}, D\left(\mathcal{A}_{\omega}\right)\right)$ in $V_{n}^{0}\left(\Omega_{f}\right) \times \mathbb{R}^{N_{c}} \times \mathbb{R}^{N_{c}}$ defined by

$$
\begin{aligned}
& D\left(\mathcal{A}_{\omega}\right)=\left\{(P v, F, f) \in V_{n}^{0}\left(\Omega_{f}\right) \times \mathbb{R}^{N_{c}} \times \mathbb{R}^{N_{c}} \mid P v-\sum_{i=1}^{N_{c}} f_{i} P D_{s}\left(\xi_{i}\right) \in D(A)\right\}, \\
& \mathcal{A}_{\omega}=\left(\begin{array}{ccc}
A+\omega I_{V_{n}^{0}} & 0 & \left((-A) P D_{s} \xi_{i}\right)_{1 \leq i \leq N_{c}} \\
0 & \omega I_{\mathbb{R}^{N_{c}}} & I_{\mathbb{R}^{N_{c}}} \\
0 & 0 & \omega I_{\mathbb{R}^{N_{c}}}
\end{array}\right),
\end{aligned}
$$

and the Dirichlet operator $D_{s} \in \mathcal{L}\left(H^{3 / 2+\ell}\left(\Gamma_{s}\right), H^{2+\ell}\left(\Omega_{f}\right)\right)$ is defined by $D_{s} \boldsymbol{\xi}=L(0, \boldsymbol{\xi})$, where the lifting operator $L$ is introduced in (2.10).

We can rewrite the system (3.4) in the form

$$
\begin{aligned}
& \frac{\mathrm{d}}{\mathrm{d} t}\left(\begin{array}{c}
P v \\
F \\
f
\end{array}\right)=\mathcal{A}_{\omega}\left(\begin{array}{c}
P v \\
F \\
f
\end{array}\right)+B h, \quad\left(\begin{array}{c}
P v(0) \\
F(0) \\
f(0)
\end{array}\right)=\left(\begin{array}{c}
P v_{0} \\
0 \\
0
\end{array}\right), \\
& (I-P) v(t)=\sum_{i=1}^{N_{c}} f_{i}(t)(I-P) D_{s} \xi_{i},
\end{aligned}
$$


where the control operator $B$ is defined by

$$
B=\left(\begin{array}{lll}
0 & 0 & 1
\end{array}\right)^{T} .
$$

We have also to extend the operator $\left(\mathcal{A}_{\omega}, D\left(\mathcal{A}_{\omega}\right)\right)$ to an unbounded operator in $V^{-2}\left(\Omega_{f}\right) \times \mathbb{R}^{N_{c}} \times \mathbb{R}^{N_{c}}$. This extension, defined by the extrapolation method, is denoted by $\left(\mathcal{A}_{\omega}, D\left(\mathcal{A}_{\omega} ; V^{-2}\left(\Omega_{f}\right) \times \mathbb{R}^{N_{c}} \times \mathbb{R}^{N_{c}}\right)\right)$, with

$$
D\left(\mathcal{A}_{\omega} ; V^{-2}\left(\Omega_{f}\right) \times \mathbb{R}^{N_{c}} \times \mathbb{R}^{N_{c}}\right)=\left\{(P v, F, f) \in V_{n}^{0}\left(\Omega_{f}\right) \times \mathbb{R}^{N_{c}} \times \mathbb{R}^{N_{c}} \mid P v-\sum_{i=1}^{N_{c}} f_{i} P D_{s}\left(\xi_{i}\right) \in V_{n}^{0}\left(\Omega_{f}\right)\right\}
$$

where $P$ is the operator introduced in Section 2.2, and

$$
\mathcal{A}_{\omega}=\left(\begin{array}{ccc}
A+\omega I_{V^{-2}}+ & 0 & \left((-A) P D_{s} \xi_{i}\right)_{1 \leq i \leq N_{c}} \\
0 & \omega I_{\mathbb{R}^{N_{c}}} & I_{\mathbb{R}^{N_{c}}} \\
0 & 0 & \omega I_{\mathbb{R}^{N_{c}}}
\end{array}\right)
$$

Remark 3.2. To prove Theorem 1.1, we study in Section 4 the nonhomogeneous closed-loop system (4.1). To prove stability results for system (4.1), in the proof of Theorem 4.1, we first use the fact that the pair $\left(\mathcal{A}_{\omega}, B\right)$ is stabilizable in $V^{-2}\left(\Omega_{f}\right) \times \mathbb{R}^{N_{c}} \times \mathbb{R}^{N_{c}}$. Thus, it seems sufficient to study the semigroup generated by $\left(\mathcal{A}_{\omega}, D\left(\mathcal{A}_{\omega} ; V^{-2}\left(\Omega_{f}\right) \times \mathbb{R}^{N_{c}} \times \mathbb{R}^{N_{c}}\right)\right)$, and the stabilizability of the pair $\left(\mathcal{A}_{\omega}, B\right)$ in $V^{-2}\left(\Omega_{f}\right) \times \mathbb{R}^{N_{c}} \times \mathbb{R}^{N_{c}}$. However, we have to recall that the operator $\left(\mathcal{A}_{\omega}, D\left(\mathcal{A}_{\omega} ; V^{-2}\left(\Omega_{f}\right) \times \mathbb{R}^{N_{c}} \times \mathbb{R}^{N_{c}}\right)\right)$ is defined, from the operator $\left(\mathcal{A}_{\omega}, D\left(\mathcal{A}_{\omega}\right)\right)$, by the extrapolation method. Moreover, it is easier to study the stabilizability of the pair $\left(\mathcal{A}_{\omega}, B\right)$ in $V_{n}^{0}\left(\Omega_{f}\right) \times \mathbb{R}^{N_{c}} \times \mathbb{R}^{N_{c}}$ rather than in $V^{-2}\left(\Omega_{f}\right) \times \mathbb{R}^{N_{c}} \times \mathbb{R}^{N_{c}}$. It is why after studying in Proposition 3.5 the stabilizability of the pair $\left(\mathcal{A}_{\omega}, B\right)$ in $V_{n}^{0}\left(\Omega_{f}\right) \times \mathbb{R}^{N_{c}} \times \mathbb{R}^{N_{c}}$, we construct a feedback $K$ in Proposition 3.6 able to stabilize the pair $\left(\mathcal{A}_{\omega}, B\right)$ both in $V_{n}^{0}\left(\Omega_{f}\right) \times \mathbb{R}^{N_{c}} \times \mathbb{R}^{N_{c}}$ and in $V^{-2}\left(\Omega_{f}\right) \times \mathbb{R}^{N_{c}} \times \mathbb{R}^{N_{c}}$. Thus the stabilizability of the pair $\left(\mathcal{A}_{\omega}, B\right)$ in $V^{-2}\left(\Omega_{f}\right) \times \mathbb{R}^{N_{c}} \times \mathbb{R}^{N_{c}}$ is obtained as a byproduct of that proposition.

Proposition 3.3. The operator $\left(\mathcal{A}_{\omega}, D\left(\mathcal{A}_{\omega}\right)\right)\left(\right.$ resp. $\left.\left(\mathcal{A}_{\omega}, D\left(\mathcal{A}_{\omega} ; V^{-2}\left(\Omega_{f}\right) \times \mathbb{R}^{N_{c}} \times \mathbb{R}^{N_{c}}\right)\right)\right)$ is the infinitesimal generator of an analytic semigroup on $V_{n}^{0}\left(\Omega_{f}\right) \times \mathbb{R}^{N_{c}} \times \mathbb{R}^{N_{c}}\left(\right.$ resp. $\left.V^{-2}\left(\Omega_{f}\right) \times \mathbb{R}^{N_{c}} \times \mathbb{R}^{N_{c}}\right)$, with a compact resolvent.

Proof. We can set

$$
A_{s}=\left(\begin{array}{cc}
\omega I_{\mathbb{R}^{N_{c}}} & I_{\mathbb{R}^{N_{c}}} \\
0 & \omega I_{\mathbb{R}^{N_{c}}}
\end{array}\right)
$$

and we can proceed as in Proof of Theorem 3.6 of [21] to prove the analiticity of the semigroup both on $V_{n}^{0}\left(\Omega_{f}\right) \times \mathbb{R}^{N_{c}} \times \mathbb{R}^{N_{c}}$ and on $\left.V^{-2}\left(\Omega_{f}\right) \times \mathbb{R}^{N_{c}} \times \mathbb{R}^{N_{c}}\right)$. The compacteness of the resolvent can be easily checked. 


\subsection{Hautus criterion}

Proposition 3.4. The adjoint of the unbounded operator $\left(\mathcal{A}_{\omega}, D\left(\mathcal{A}_{\omega}\right)\right)$ in $V_{n}^{0}\left(\Omega_{f}\right) \times \mathbb{R}^{N_{c}} \times \mathbb{R}^{N_{c}}$ is defined by

$$
D\left(\mathcal{A}_{\omega}^{*}\right)=\left(V^{2}\left(\Omega_{f}\right) \cap V_{0}^{1}\left(\Omega_{f}\right)\right) \times \mathbb{R}^{N_{c}} \times \mathbb{R}^{N_{c}}
$$

and

$$
\mathcal{A}_{\omega}^{*}=\left(\begin{array}{ccc}
A+\omega I & 0 & 0 \\
0 & \omega I_{\mathbb{R}^{N_{c}}} & 0 \\
\left(\left(\sigma\left(\cdot, N_{\psi}(\cdot)\right) n, \xi_{i}\right)_{\Gamma_{s}}\right)_{1 \leq i \leq N_{c}} & I_{\mathbb{R}^{N_{c}}} & \omega I_{\mathbb{R}^{N_{c}}}
\end{array}\right),
$$

where $\left(\sigma\left(\phi, N_{\psi}(\phi)\right) n, \xi_{i}\right)_{\Gamma_{s}}$ stands for $\int_{\Gamma_{s}} \sigma\left(\phi, N_{\psi}(\phi)\right) n \cdot \xi_{i} \mathrm{~d} x$, and, for any $\phi \in V^{2}\left(\Omega_{f}\right) \cap V_{0}^{1}\left(\Omega_{f}\right)$, $N_{\psi}(\phi)=$ $\psi \in H^{1}\left(\Omega_{f}\right) / \mathbb{R}$ is the pressure satisfying

$$
-\Delta \psi=0 \quad \text { in } \Omega_{f}, \quad \frac{\partial \psi}{\partial n}=\nu \Delta \phi \cdot n \quad \text { on } \Gamma_{s} \cup \Gamma_{e}
$$

Proof. The proof can be done in a classical way and is left to the reader.

Proposition 3.5. The pair $\left(\mathcal{A}_{\omega}, B\right)$ is stabilizable in $V_{n}^{0}\left(\Omega_{f}\right) \times \mathbb{R}^{N_{c}} \times \mathbb{R}^{N_{c}}$.

Proof. Step 1. We first assume that

$$
-\omega \notin \sigma(A)
$$

It is sufficient to verify the Hautus test, see, e.g., Proposition 1.3 of [26]. We have to consider the adjoint eigenvalue problem

$$
\begin{aligned}
& \lambda \in \mathbb{C}, \\
& \lambda \phi-\operatorname{div} \sigma(\phi, \psi)-\omega \phi=0, \quad \text { in } \Omega_{f}, \\
& \operatorname{div} \phi=0 \quad \text { in } \Omega_{f}, \\
& \phi=0 \text { on } \Gamma_{s}, \quad \phi=0 \text { on } \Gamma_{e}, \\
& \lambda \Xi=\omega \Xi, \\
& \lambda \zeta=\omega \zeta+\Xi+\left(\left(\sigma(\phi, \psi) n, \xi_{i}\right)_{\Gamma_{s}}\right)_{1 \leq i \leq N_{c}} .
\end{aligned}
$$

We want to show that if $(\lambda, \phi, \psi, \Xi, \zeta)$ is a solution to system (3.7) with $\lambda$ such that $\operatorname{Re} \lambda \geq 0$, and if

$$
B^{*}(\phi, \Xi, \zeta)=\zeta=0
$$

then $(\phi, \Xi)=(0,0)$. We have to consider two different cases.

Case 1. If $\lambda=\omega$, from condition (3.6) and from $(3.7)_{1-3}$, it follows that $(\phi, \psi)=(0,0)$. In particular, we have $\left.\sigma(\phi, \psi) n\right|_{\Gamma_{s}}=0$. With $(3.7)_{5}$ and the condition $\sigma(\phi, \psi) n=0$ on $\Gamma_{s}$, we deduce that $\Xi=0$ and the proof is complete in that case.

Case 2. We consider the case when $\lambda \neq \omega$. Thus, $(\phi, \psi)$ is a pair solution to the eigenvalue problem for the Stokes operator with Dirichlet boundary conditions associated with the eigenvalue $\lambda-\omega$. Since $\lambda \neq \omega$, from $(3.7)_{4}$ it follows that $\Xi=0$. With $(3.7)_{5}$, it follows that $\left(\left(\sigma(\phi, \psi) n, \xi_{i}\right)_{\Gamma_{s}}\right)_{1 \leq i \leq N_{c}}=\mathbf{0}_{\mathbb{R}^{N_{c}}}$. Due to (3.3), we have 
$\sigma(\phi, \psi) n-\frac{n}{\left|\Gamma_{s}\right|} \int_{\Gamma_{s}} \sigma(\phi, \psi) n \cdot n \mathrm{~d} x=0$ on $\Gamma_{s}$, and the unique continuation results for the Stokes equation with Dirichlet boundary conditions gives $\phi=0$.

Step 2. If (3.6) is not satisfied, that is if $-\omega \in \sigma(A)$, we choose $-\widetilde{\omega}<-\omega$, close enough to $-\omega$, satisfying $-\widetilde{\omega} \notin \sigma(A)$ and $[-\widetilde{\omega},-\omega[\cap \sigma(A)=\emptyset$. Since $-\widetilde{\omega} \notin \sigma(A)$ and $[-\widetilde{\omega},-\omega[\cap \sigma(A)=\emptyset$, as in Step 1, we can show that the pair $\left(\mathcal{A}_{\omega}+(\widetilde{\omega}-\omega) I, B\right)$ is stabilizable in $V_{n}^{0}\left(\Omega_{f}\right) \times \mathbb{R}^{N_{c}} \times \mathbb{R}^{N_{c}}$. Indeed, we have

$\{\phi \in D(A) \mid(\phi, \lambda-\omega)$ is a pair of eigenfunction-eigenvalue of the Stokes operator with $\lambda \geq 0\}$

$=\{\phi \in D(A) \mid(\phi, \lambda-\widetilde{\omega})$ is a pair of eigenfunction-eigenvalue of the Stokes operator with $\lambda \geq 0\}$.

If $K \in \mathcal{L}\left(V_{n}^{0}\left(\Omega_{f}\right) \times \mathbb{R}^{N_{c}} \times \mathbb{R}^{N_{c}}, \mathbb{R}^{N_{c}}\right)$ is a feedback operator such that $\left(e^{t\left(\mathcal{A}_{\omega}+(\widetilde{\omega}-\omega) I+B K\right)}\right)_{t>0}$ is exponentially stable on $V_{n}^{0}\left(\Omega_{f}\right) \times \mathbb{R}^{N_{c}} \times \mathbb{R}^{N_{c}}$, then $\left(e^{t\left(\mathcal{A}_{\omega}+B K\right)}\right)_{t \geq 0}$ is also exponentially stable on $V_{n}^{0}\left(\Omega_{f}\right) \times \mathbb{R}^{N_{c}} \times \mathbb{R}^{N_{c}}$. The proof is complete.

Proposition 3.6. There exists a feedback operator $K \in \mathcal{L}\left(V_{n}^{0}\left(\Omega_{f}\right) \times \mathbb{R}^{N_{c}} \times \mathbb{R}^{N_{c}}, \mathbb{R}^{N_{c}}\right) \cap \mathcal{L}\left(V^{-2}\left(\Omega_{f}\right) \times \mathbb{R}^{N_{c}} \times\right.$ $\left.\mathbb{R}^{N_{c}}, \mathbb{R}^{N_{c}}\right)$ such that $\left(\mathcal{A}_{\omega}+B K, \mathcal{D}\left(\mathcal{A}_{\omega}\right)\right.$ (resp. $\left(\mathcal{A}_{\omega}+B K, \mathcal{D}\left(\mathcal{A}_{\omega} ; V^{-2}\left(\Omega_{f}\right) \times \mathbb{R}^{N_{c}} \times \mathbb{R}^{N_{c}}\right)\right.$ ) is the infinitesimal generator of an analytic and exponentially stable semigroup on $V_{n}^{0}\left(\Omega_{f}\right) \times \mathbb{R}^{N_{c}} \times \mathbb{R}^{N_{c}}$ (resp. $V^{-2}\left(\Omega_{f}\right) \times \mathbb{R}^{N_{c}} \times$ $\left.\mathbb{R}^{N_{c}}\right)$.

Proof. It is sufficient to apply the strategy developed in [20] for the Oseen equations. In [20], feedbacks are obtained by looking for feedback controls stabilizing the Oseen system projected onto the finite dimensional unstable subspace of the Oseen operator. Here, we have to find feedback controls stabilizing the extended system (3.4) projected onto the finite dimensional unstable subspace of $\mathcal{A}_{\omega}$, that we denote by $Z_{u}$. If $P_{u}$ is the projector in $V_{n}^{0}\left(\Omega_{f}\right) \times \mathbb{R}^{N_{c}} \times \mathbb{R}^{N_{c}}$ onto $Z_{u}$ satisfying $P_{u} \mathcal{A}_{\omega}=\mathcal{A}_{\omega} P_{u}$, this projector can be extended as a projector from $V^{-2}\left(\Omega_{f}\right) \times \mathbb{R}^{N_{c}} \times \mathbb{R}^{N_{c}}$ onto $Z_{u}$ (see [24], Lem. 3.17). Because of that property, if $K_{u}$ belongs to $\mathcal{L}\left(Z_{u}, \mathbb{R}^{N_{c}}\right)$, the operator $K=K_{u} P_{u}$ belongs to $\mathcal{L}\left(V_{n}^{0}\left(\Omega_{f}\right) \times \mathbb{R}^{N_{c}} \times \mathbb{R}^{N_{c}}, \mathbb{R}^{N_{c}}\right)$ and also $\mathcal{L}\left(V^{-2}\left(\Omega_{f}\right) \times \mathbb{R}^{N_{c}} \times \mathbb{R}^{N_{c}}, \mathbb{R}^{N_{c}}\right)$. If in

addition, $K_{u}$ is a feedback stabilizing exponentially the pair $\left(P_{u} \mathcal{A}_{\omega}, P_{u} B\right)$ in $Z_{u}$, that is if $\left(e^{t\left(P_{u} \mathcal{A}_{\omega}+P_{u} B K_{u}\right)}\right)_{t \geq 0}$ is exponentially stable on $Z_{u}$, then $\left(e^{t\left(\mathcal{A}_{\omega}+B K\right)}\right)_{t \geq 0}$ is exponentially stable both on $V_{n}^{0}\left(\Omega_{f}\right) \times \mathbb{R}^{N_{c}} \times \mathbb{R}^{N_{c}}$ and on $V^{-2}\left(\Omega_{f}\right) \times \mathbb{R}^{N_{c}} \times \mathbb{R}^{N_{c}}$. The fact that $\left(\mathcal{A}_{\omega}+B K, \mathcal{D}\left(\mathcal{A}_{\omega}\right)\right)$ is the infinitesimal generator of an analytic semigroup on $V_{n}^{0}\left(\Omega_{f}\right) \times \mathbb{R}^{N_{c}} \times \mathbb{R}^{N_{c}}$ follows from the fact that $B K$ is a bounded perturbation of $\left(\mathcal{A}_{\omega}, \mathcal{D}\left(\mathcal{A}_{\omega}\right)\right)$. The same argument is valid for the extension of the operator $\mathcal{A}_{\omega}$ in $V^{-2}\left(\Omega_{f}\right) \times \mathbb{R}^{N_{c}} \times \mathbb{R}^{N_{c}}$.

This approach, used in [20], has been first introduced in [24]. Another approach for constructing such feedbacks is introduced in [22] for the 3D Oseen system.

\section{Closed-Loop Nonhomogeneous Linear System}

We want to prove regularity results for the system

$$
\begin{aligned}
& \frac{\partial v}{\partial t}-\omega v-\nu \Delta v+\nabla q=\mathcal{F} \quad \text { and } \quad \operatorname{div} v=\mathcal{G}=\operatorname{div} \mathbf{g} \text { in } Q_{f}^{\infty}, \\
& v(0)=v_{0} \text { in } \Omega_{f}, \\
& v=\sum_{i=1}^{N_{c}} f_{i}(t) \xi_{i}(y)+n \delta \quad \text { on } \Sigma_{s}^{\infty}, \quad v=0 \text { on } \Sigma_{0}^{\infty}, \\
& F^{\prime}=\omega F+f, \quad F(0)=0, \\
& f^{\prime}=\omega f+K(P v, F, f), \quad f(0)=0,
\end{aligned}
$$


where $K$ is the feedback operator introduced in Proposition 3.6, and the function $\delta$ defined by

$$
\delta(t)=\left|\Gamma_{s}\right|^{-1} \int_{\Gamma_{s}} \mathbf{g}(t) \cdot n \mathrm{~d} x
$$

is added to satisfy the compatibility condition

$$
\int_{\Gamma_{s}} v \cdot n \mathrm{~d} x=\int_{\Gamma_{s}} \mathrm{~g} \cdot n \mathrm{~d} x
$$

As in [23], we can prove that $(v, F, f)$ is solution to (4.1) if and only if it satisfies

$$
\begin{aligned}
& \frac{\mathrm{d}}{\mathrm{d} t}\left(\begin{array}{c}
P v \\
F \\
f
\end{array}\right)=\left(\mathcal{A}_{\omega}+B K\right)\left(\begin{array}{c}
P v \\
F \\
f
\end{array}\right)+\left(\begin{array}{c}
\mathcal{F}+(-A) L(\mathcal{G}, n \delta) \\
0 \\
0
\end{array}\right), \quad\left(\begin{array}{c}
P v(0) \\
F(0) \\
f(0)
\end{array}\right)=\left(\begin{array}{c}
P v_{0} \\
0 \\
0
\end{array}\right), \\
& (I-P) v(t)=\sum_{i=1}^{N_{c}} f_{i}(I-P) D_{s} \xi_{i}+(I-P) L(\mathcal{G}, n \delta) .
\end{aligned}
$$

Theorem 4.1. Assume that $\mathcal{F}, v_{0}, \mathbf{g}$, and $\mathcal{G}$ obey the assumptions (2.5) and (2.6) of Theorem 2.3 with $\boldsymbol{\xi}=$ $\sum_{i=1}^{N_{c}} f_{i}(t) \xi_{i}(y)+n \delta$.

Then the system (4.1) admits a unique solution $(v, q, F, f) \in E_{\ell} \times P_{\ell} \times H^{3+\ell / 2}\left(0, \infty ; \mathbb{R}^{N_{c}}\right) \times$ $H^{2+\ell / 2}\left(0, \infty ; \mathbb{R}^{N_{c}}\right)$, moreover we have the following estimates

$$
\begin{aligned}
& \|v\|_{E_{\ell}}+\|\nabla q\|_{F_{\ell}}+\|F\|_{H^{3+\ell / 2}\left(0, \infty ; \mathbb{R}^{N_{c}}\right)}+\|f\|_{H^{2+\ell / 2}\left(0, \infty ; \mathbb{R}^{N_{c}}\right)} \\
& \leq C_{1}\left(\left\|v_{0}\right\|_{H^{1+\ell}\left(\Omega_{f}\right)}+\|\mathcal{F}\|_{F_{\ell}}+\|\mathbf{g}\|_{\mathbf{G}_{\ell}}+\|\mathcal{G}\|_{G_{\ell}}+\|\delta\|_{H^{1+\ell / 2}(0, \infty)}\right) .
\end{aligned}
$$

Proof. Step 1. Estimate in $L^{2}\left(0, \infty ; V_{n}^{0}\left(\Omega_{f}\right) \times \mathbb{R}^{N_{c}} \times \mathbb{R}^{N_{c}}\right)$. As $\mathcal{G}$ belongs to $L^{2}\left(0, \infty ; H^{1+\ell}\left(\Omega_{f}\right)\right.$, then $L(\mathcal{G}, \delta n)$ belongs to $L^{2}\left(0, \infty ; H^{2+\ell}\left(\Omega_{f}\right)\right.$, and $\mathcal{F}+(-A) P L(\mathcal{G}, \delta n)$ belongs to $L^{2}\left(0, \infty ; V^{-2}\left(\Omega_{f}\right)\right)$. Thus, since $\left(e^{t\left(\mathcal{A}_{\omega}+B K\right)}\right)_{t>0}$ is exponentially stable on $V^{-2}\left(\Omega_{f}\right) \times \mathbb{R}^{N_{c}} \times \mathbb{R}^{N_{c}}$, we can apply ([2], II-1, Thm. 3.1) and we have

$$
\begin{aligned}
& \|P v\|_{L^{2}\left(0, \infty ; V_{n}^{0}\left(\Omega_{f}\right)\right)}+\|F\|_{L^{2}\left(0, \infty ; \mathbb{R}^{N_{c}}\right)}+\|f\|_{L^{2}\left(0, \infty ; \mathbb{R}^{N_{c}}\right)} \\
& \leq C\left(\left\|v_{0}\right\|_{V^{-1}\left(\Omega_{f}\right)}+\|\mathcal{F}\|_{L^{2}\left(0, \infty ; V^{-2}\left(\Omega_{f}\right)\right)}+\|(-A) P L(\mathcal{G}, \delta n)\|_{L^{2}\left(0, \infty ; V^{-2}\left(\Omega_{f}\right)\right)}\right) .
\end{aligned}
$$

The estimate of $(I-P) v$ is

$$
\|(I-P) v\|_{L^{2}\left(0, \infty ; H^{2+\ell}\left(\Omega_{f} ; \mathbb{R}^{3}\right)\right)} \leq C\left(\|f\|_{L^{2}\left(0, \infty ; \mathbb{R}^{\left.N_{c}\right)}\right.}+\|\mathcal{G}\|_{L^{2}\left(0, \infty ; H^{1+\ell}\left(\Omega_{f}\right)\right.}+\|\delta\|_{L^{2}(0, \infty)}\right) .
$$

With Theorem 2.3, (4.4), and (4.5), we obtain

$$
\begin{aligned}
& \|v\|_{L^{2}\left(0, \infty ; L^{2}\left(\Omega_{f} ; \mathbb{R}^{3}\right)\right)}+\|F\|_{L^{2}\left(0, \infty ; \mathbb{R}^{N_{c}}\right)}+\|f\|_{L^{2}\left(0, \infty ; \mathbb{R}^{N_{c}}\right)} \\
& \leq C\left(\left\|v_{0}\right\|_{V^{-1}\left(\Omega_{f}\right)}+\|\mathcal{F}\|_{L^{2}\left(0, \infty ; V^{-2}\left(\Omega_{f}\right)\right)}+\|(-A) P L(\mathcal{G}, \delta n)\|_{L^{2}\left(0, \infty ; V^{-2}\left(\Omega_{f}\right)\right)}\right) .
\end{aligned}
$$


Therefore, taking into account the equations satisfied by $F$ and $f$, we also have

$$
\begin{aligned}
& \|v\|_{L^{2}\left(0, \infty ; L^{2}\left(\Omega_{f} ; \mathbb{R}^{3}\right)\right)}+\|F\|_{H^{2}\left(0, \infty ; \mathbb{R}^{N_{c}}\right)}+\|f\|_{H^{1}\left(0, \infty ; \mathbb{R}^{N_{c}}\right)} \\
& \leq C\left(\left\|v_{0}\right\|_{V^{-1}\left(\Omega_{f}\right)}+\|\mathcal{F}\|_{L^{2}\left(0, \infty ; V^{-2}\left(\Omega_{f}\right)\right)}+\|(-A) P L(\mathcal{G}, \delta n)\|_{L^{2}\left(0, \infty ; V^{-2}\left(\Omega_{f}\right)\right)}\right) .
\end{aligned}
$$

Step 2. Improved regularity. Now, we rewrite the first equation of system (4.1) in the form

$$
\frac{\partial v}{\partial t}-\nu \Delta v+\nabla q=\mathcal{F}+\omega v \quad \text { and } \quad \operatorname{div} v=\mathcal{G}=\operatorname{div} \mathbf{g} \quad \text { in } Q_{f}^{\infty} .
$$

From (4.7), with the first equation of system (4.1) written in the form (4.8), using regularity results for the Stokes equations ([23], Thm. 5.5), we first obtain

$$
\|v\|_{H^{2,1}\left(Q_{f}^{\infty}\right)} \leq C\left(\left\|v_{0}\right\|_{V_{0}^{1}\left(\Omega_{f}\right)}+\|\mathcal{F}\|_{L^{2}\left(0, \infty ; L^{2}\left(\Omega_{f}\right)\right)}+\|\mathcal{G}\|_{H^{1}\left(0, \infty ; H^{1}\left(\Omega_{f}\right)\right)}+\|\delta\|_{H^{1}(0, \infty)}\right) .
$$

Knowing that $v \in H^{2,1}\left(Q_{f}^{\infty}\right), F \in H^{2}\left(0, \infty ; \mathbb{R}^{N_{c}}\right)$, and $f \in H^{1}\left(0, \infty ; \mathbb{R}^{N_{c}}\right)$, we have that $K(v, F, f)$ belongs to $H^{1}\left(0, \infty ; \mathbb{R}^{N_{c}}\right)$. Thus, $f$ belongs to $H^{2}\left(0, \infty ; \mathbb{R}^{N_{c}}\right)$ and $F$ belongs to $H^{3}\left(0, \infty ; \mathbb{R}^{N_{c}}\right)$. Still using regularity results for the Stokes equations stated in Theorem 2.3, we obtain

$$
\begin{aligned}
& \|v\|_{E_{\ell}}+\|\nabla q\|_{F_{\ell}}+\|F\|_{H^{3}\left(0, \infty ; \mathbb{R}^{N_{c}}\right)}+\|f\|_{H^{2}\left(0, \infty ; \mathbb{R}^{N_{c}}\right)} \\
& \leq C_{1}\left(\left\|v_{0}\right\|_{H^{1+\ell}\left(\Omega_{f}\right)}+\|\mathcal{F}\|_{F_{\ell}}+\|\mathbf{g}\|_{\mathbf{G}_{\ell}}+\|\mathcal{G}\|_{G_{\ell}}\right) .
\end{aligned}
$$

From this estimate, we deduce that $K(v, F, f)$ belongs to $H^{1+\ell / 2}\left(0, \infty ; \mathbb{R}^{N_{c}}\right)$, and next that $f$ belongs to $H^{2+\ell / 2}\left(0, \infty ; \mathbb{R}^{N_{c}}\right), F$ belongs to $H^{2+\ell / 2}\left(0, \infty ; \mathbb{R}^{N_{c}}\right)$, and that $(4.3)$ is satisfied.

\section{Estimates of the nONLinear terms over the time interval $(0, \infty)$}

\subsection{Estimates of $\widehat{X}$ and $\widehat{Y}$}

In addition to the spaces $E_{\ell}, F_{\ell}$, and $P_{\ell}$ already introduced before, we also need

$$
\begin{aligned}
S_{\ell}=\left\{f \in L^{\infty}\left(0, \infty ; H^{1+\ell}\left(\Omega_{f}\right)\right) \cap H_{\mathrm{loc}}^{1}\left([0, \infty) ; H^{1+\ell}\left(\Omega_{f}\right)\right)\right. \\
\left.\mid \partial_{t} f \in L^{2}\left(0, \infty ; H^{1+\ell}\left(\Omega_{f}\right)\right) \cap H^{\ell / 2+1 / 2}\left(0, \infty ; L^{2}\left(\Omega_{f}\right)\right)\right\},
\end{aligned}
$$

and

$$
\begin{aligned}
D_{\ell}=\left\{f \in L^{\infty}\left(0, \infty ; H^{\ell}\left(\Omega_{f}\right)\right) \cap H_{\mathrm{loc}}^{1}\left([0, \infty) ; H^{\ell}\left(\Omega_{f}\right)\right)\right. \\
\left.\mid \partial_{t} f \in L^{2}\left(0, \infty ; H^{\ell}\left(\Omega_{f}\right)\right) \cap H^{\ell / 2}\left(0, \infty ; L^{2}\left(\Omega_{f}\right)\right)\right\} .
\end{aligned}
$$

The spaces $S_{\ell}, D_{\ell}$, and $I_{\ell}$ (defined in Thm. 1.1) will be equipped with the norms

$$
\begin{aligned}
& \|f\|_{S_{\ell}} \\
& =\|f\|_{L^{\infty}\left(0, \infty ; H^{1+\ell}\left(\Omega_{f}\right)\right)}+\left\|\partial_{t} f\right\|_{L^{2}\left(0, \infty ; H^{1+\ell}\left(\Omega_{f}\right)\right)}+\left\|\partial_{t} f\right\|_{H^{\ell / 2+1 / 2}\left(0, \infty ; L^{2}\left(\Omega_{f}\right)\right)}, \\
& \|f\|_{D_{\ell}}=\|f\|_{L^{\infty}\left(0, \infty ; H^{\ell}\left(\Omega_{f}\right)\right)}+\left\|\partial_{t} f\right\|_{L^{2}\left(0, \infty ; H^{\ell}\left(\Omega_{f}\right)\right)}+\left\|\partial_{t} f\right\|_{H^{\ell / 2}\left(0, \infty ; L^{2}\left(\Omega_{f}\right)\right)}
\end{aligned}
$$

and

$$
\|f\|_{I_{\ell}}=\|f\|_{L^{\infty}\left(0, \infty ; H^{2+\ell}\left(\Omega_{f}\right)\right)}+\left\|\partial_{t} f\right\|_{L^{2}\left(0, \infty ; H^{2+\ell}\left(\Omega_{f}\right)\right)}+\left\|\partial_{t} f\right\|_{H^{1}\left(0, \infty ; H^{\ell / 2}\left(\Omega_{f}\right)\right)} .
$$


Notice that if $\widehat{u}$ belongs to $E_{\ell}$, then $\widehat{X}(y, t)=y+\int_{0}^{t} e^{-\omega s} \widehat{u}(y, s) \mathrm{d} s$ belongs to $I_{\ell}$, and that

$$
\|\widehat{X}\|_{I_{\ell}} \leq C\left(1+\|\widehat{u}\|_{S_{\ell}}\right) .
$$

We are now going to estimate

$$
J_{\widehat{X}}(y, t)=I+\int_{0}^{t} e^{-\omega s} \nabla \widehat{u}(y, s) \mathrm{d} s
$$

Lemma 5.1. Let $0<\gamma<1$ and $\omega>0$ be chosen. Let us assume that

$$
\|\widehat{u}\|_{L^{2}\left(0, \infty ; H^{2+\ell}\left(\Omega_{f}\right)\right)} \leq \frac{\gamma \sqrt{2 \omega}}{C\left(H^{1+\ell} ; L^{\infty}\right)}
$$

where $C\left(H^{1+\ell} ; L^{\infty}\right)$ is the constant of the imbedding $H^{1+\ell}\left(\Omega_{f}\right) \hookrightarrow L^{\infty}\left(\Omega_{f}\right)$. Then, the mapping

$$
\widehat{X}(y, t)=y+\int_{0}^{t} e^{-\omega \tau} \widehat{u}(y, \tau) \mathrm{d} \tau
$$

obeys

$$
\left\|J_{\widehat{X}}-I\right\|_{C\left(\overline{Q_{f}^{\infty}}\right)} \leq \gamma<1
$$

Proof. We have

$$
\begin{aligned}
& \left\|J_{\widehat{X}}(\cdot, t)-I\right\|_{C\left(\overline{Q_{f}^{\infty}}\right)} \leq C\left(H^{1+\ell} ; L^{\infty}\right)\left\|J_{\widehat{X}}(\cdot, t)-I\right\|_{L^{\infty}\left(0, \infty ; H^{1+\ell}\left(\Omega_{f}\right)\right)} \\
& \leq C\left(H^{1+\ell} ; L^{\infty}\right) \int_{0}^{\infty} e^{-\omega \tau}\|\widehat{u}(\cdot, \tau)\|_{H^{2+\ell}\left(\Omega_{f}\right)} \mathrm{d} \tau \\
& \leq \frac{C\left(H^{1+\ell} ; L^{\infty}\right)}{\sqrt{2 \omega}}\|\widehat{u}(\cdot, \tau)\|_{L^{2}\left(0, \infty ; H^{2+\ell}\left(\Omega_{f}\right)\right)} \leq \gamma
\end{aligned}
$$

Lemma 5.2. If $\widehat{u}$ belongs to $E_{\ell}$, then the mapping $J_{\widehat{X}}-I$ belongs to $S_{\ell}$. And there exists a constant $C(\omega)$ such that

$$
\left\|J_{\widehat{X}}(\cdot, t)-I\right\|_{S_{\ell}} \leq C(\omega)\|\widehat{u}\|_{E_{\ell}} .
$$

Proof. We estimate $J_{\widehat{X}}-I$ in $L^{\infty}\left(0, \infty ; H^{1+\ell}\left(\Omega_{f}\right)\right)$ as follows:

$$
\begin{aligned}
& \left\|J_{\widehat{X}}(\cdot, t)-I\right\|_{L^{\infty}\left(0, \infty ; H^{1+\ell}\left(\Omega_{f}\right)\right)} \\
& \leq \int_{0}^{t} e^{-\omega \tau}\|\widehat{u}(\cdot, \tau)\|_{H^{2+\ell}\left(\Omega_{f}\right)} \mathrm{d} \tau \leq \frac{1}{\sqrt{2 \omega}}\|\widehat{u}\|_{L^{2}\left(0, \infty ; H^{2+\ell}\left(\Omega_{f}\right)\right)} .
\end{aligned}
$$

We have $\partial_{t} J_{\widehat{X}}(y, t)=e^{-\omega t} \nabla \widehat{u}(y, t)$. Since

$$
\|\nabla \widehat{u}\|_{H^{1+\ell, 1 / 2+\ell / 2}\left(Q_{f}^{\infty}\right)} \leq\|\widehat{u}\|_{E_{\ell}},
$$


the proof is complete.

Lemma 5.3. If $\widehat{u}$ belongs to $E_{\ell}$, and if

$$
C(\omega)\|\widehat{u}\|_{E_{\ell}} \leq \gamma<1,
$$

then $J_{\widehat{Y}} \in S_{\ell}$ and

$$
\left\|I-J_{\widehat{Y}}\right\|_{S_{\ell}} \leq \frac{\gamma}{1-\gamma} .
$$

Proof. Since $J_{\widehat{Y}}=J_{\widehat{X}}^{-1}$, with Lemma A.1, we have

$$
I-J_{\widehat{Y}}=-\sum_{k=1}^{\infty}\left(I-J_{\widehat{X}}\right)^{k}
$$

Thus

$$
\left\|I-J_{\widehat{Y}}\right\|_{S_{\ell}} \leq \sum_{k=1}^{\infty}\left\|I-J_{\widehat{X}}\right\|_{S_{\ell}}^{k}=\frac{\gamma}{\gamma-1} .
$$

For all $\mu$ satisfying

$$
0<\mu \leq \min \left\{\gamma / C(\omega), \gamma \sqrt{2 \omega} / C\left(H^{1+\ell} ; L^{\infty}\right)\right\}
$$

we set

$$
E_{\ell, \mu}=\left\{u \in E_{\ell} \mid\|u\|_{E_{\ell}} \leq \mu\right\} .
$$

We denote by $\mathcal{P}_{0}^{+}$the set of polynomials $f$ with nonnegative coefficients and satisfying $f(0)=0$.

Lemma 5.4. There exists a polynomial $K_{1} \in \mathcal{P}_{0}^{+}$such that, for all $\mu>0$ satisfying (5.1), all $\widehat{u} \in E_{\ell, \mu}$, we have

$$
\begin{array}{ll}
\left\|I-\operatorname{cof} J_{\widehat{X}}\right\|_{S_{\ell}} \leq K_{1}(\mu), & \left\|1-\frac{1}{\operatorname{det} J_{\widehat{X}}}\right\|_{S_{\ell}} \leq K_{1}(\mu), \\
\left\|1-\operatorname{det} J_{\widehat{X}}\right\|_{S_{\ell}} \leq K_{1}(\mu), & \left\|1-\left(\operatorname{det} J_{\widehat{X}}\right) J_{\widehat{Y}}\right\|_{S_{\ell}} \leq K_{1}(\mu), \\
\left\|I-J_{\widehat{Y}}\right\|_{S_{\ell}} \leq K_{1}(\mu), & \left\|\frac{\partial^{2} \widehat{Y}}{\partial x_{j} \partial x_{k}}\right\|_{D_{\ell}} \leq K_{1}(\mu), \\
\left\|I-\left(\operatorname{det} J_{\widehat{X}}\right) J_{\widehat{Y}}^{T}\right\|_{S_{\ell}} \leq K_{1}(\mu), & \\
\left\|\frac{\partial \widehat{Y}}{\partial t}\right\|_{S_{\ell}} \leq K_{1}(\mu) .
\end{array}
$$

Proof. The proof follows from Lemmas 5.2, 5.3, A.1, A.5, and from the following identities 


$$
\frac{\partial J_{\widehat{Y}}}{\partial t}=-J_{\widehat{X}}^{-1} \frac{\partial J_{\widehat{X}}}{\partial t} J_{\widehat{X}}^{-1}=-J_{\widehat{Y}} e^{-\omega t} \widehat{u} J_{\widehat{Y}}
$$

and

$$
\frac{\partial \widehat{Y}}{\partial t}(\widehat{X}(\cdot, \cdot), \cdot)=-J_{\widehat{Y}} \frac{\partial \widehat{X}}{\partial t}=-J_{\widehat{Y}} e^{-\omega t} \widehat{u}
$$

We can also establish the following Lipschitz estimates.

Lemma 5.5. There exists a polynomial $K_{2} \in \mathcal{P}_{0}^{+}$such that, for all $\mu>0$ satisfying (5.1), all $\widehat{u}^{1} \in E_{\ell, \mu}$, and all $\widehat{u}^{2} \in E_{\ell, \mu}$, we have

$$
\begin{aligned}
& \left\|J_{\widehat{X}^{1}}-J_{\widehat{X}^{2}}\right\|_{S_{\ell}} \leq K_{2}(\mu)\left\|\widehat{u}^{1}-\widehat{u}^{2}\right\|_{E_{\ell}}, \\
& \left\|\operatorname{cof} J_{\widehat{X}^{1}}-\operatorname{cof} J_{\widehat{X}^{2}}\right\|_{S_{\ell}} \leq K_{2}(\mu)\left\|\widehat{u}^{1}-\widehat{u}^{2}\right\|_{E_{\ell}}, \\
& \left\|\operatorname{det} J_{\widehat{X}^{1}}-\operatorname{det} J_{\widehat{X}^{2}}\right\|_{S_{\ell}} \leq K_{2}(\mu)\left\|\widehat{u}^{1}-\widehat{u}^{2}\right\|_{E_{\ell}}, \\
& \left\|\frac{1}{\operatorname{det} J_{\widehat{X}^{1}}}-\frac{1}{\operatorname{det} J_{\widehat{X}^{2}}}\right\|_{S_{\ell}} \leq K_{2}(\mu)\left\|\widehat{u}^{1}-\widehat{u}^{2}\right\|_{E_{\ell}}, \\
& \left\|J_{\widehat{Y}^{1}}-J_{\widehat{Y}^{2}}\right\|_{S_{\ell}} \leq K_{2}(\mu)\left\|\widehat{u}^{1}-\widehat{u}^{2}\right\|_{E_{\ell}}, \\
& \left\|\operatorname{det} J_{\widehat{X}^{1}} J_{\widehat{Y}^{1}}-\operatorname{det} J_{\widehat{X}^{2}} J_{\widehat{Y}^{2}}\right\|_{S_{\ell}} \leq K_{2}(\mu)\left\|\widehat{u}^{1}-\widehat{u}^{2}\right\|_{E_{\ell}}, \\
& \left\|\frac{\partial^{2} \widehat{Y}^{1}}{\partial x_{j} \partial x_{k}}-\frac{\partial^{2} \widehat{Y}^{2}}{\partial x_{j} \partial x_{k}}\right\|_{D_{\ell}} \leq K_{2}(\mu)\left\|\widehat{u}^{1}-\widehat{u}^{2}\right\|_{E_{\ell}}, \\
& \left\|\frac{\partial \widehat{Y}^{1}}{\partial t}-\frac{\partial \widehat{Y}^{2}}{\partial t}\right\|_{S_{\ell}} \leq K_{2}(\mu)\left\|\widehat{u}^{1}-\widehat{u}^{2}\right\|_{E_{\ell}}, \\
& \left\|\frac{\partial J_{\widehat{Y}^{1}}}{\partial t}-\frac{\partial J_{\widehat{Y}^{2}}}{\partial t}\right\|_{S_{\ell}} \leq K_{2}(\mu)\left\|\widehat{u}^{1}-\widehat{u}^{2}\right\|_{E_{\ell}},
\end{aligned}
$$

where $\widehat{X}^{1}(y, t)=y+\int_{0}^{t} e^{-\omega s} \widehat{u}^{1}(y, s) \mathrm{d} s, \widehat{X}^{2}(y, t)=y+\int_{0}^{t} e^{-\omega s} \widehat{u}^{2}(y, s) \mathrm{d} s, \widehat{Y}^{1}(\cdot, t)$ is the inverse of $\widehat{X}^{1}(\cdot, t)$, and $\widehat{Y}^{2}(\cdot, t)$ is the inverse of $\widehat{X}^{2}(\cdot, t)$.

\subsection{Lipschitz estimate on $\mathcal{F}$}

For all $\mu$ satisfying (5.1), we set

$$
P_{\ell, \mu}=\left\{p \in P_{\ell} \mid\|\nabla p\|_{F_{\ell}} \leq \mu\right\} .
$$

Lemma 5.6. There exists a polynomial $C_{\mathcal{F}} \in \mathcal{P}_{0}^{+}$such that, for all $\mu>0$ satisfying (5.1), all $\widehat{u} \in E_{\ell, \mu}$, all $\widehat{p} \in P_{\ell, \mu}$, all $\widehat{u}^{1} \in E_{\ell, \mu}$, all $\widehat{p}_{1} \in P_{\ell, \mu}$, all $\widehat{u}^{2} \in E_{\ell, \mu}$, and all $\widehat{p}_{2} \in P_{\ell, \mu}$, we have

$$
\|\mathcal{F}(\widehat{u}, \widehat{p})\|_{F_{\ell}} \leq \mu C_{\mathcal{F}}(\mu),
$$


and

$$
\begin{aligned}
& \left\|\mathcal{F}_{1}\left(\widehat{u}^{1}\right)-\mathcal{F}_{1}\left(\widehat{u}^{2}\right)\right\|_{F_{\ell}}+\left\|\mathcal{F}_{2}\left(\widehat{u}^{1}\right)-\mathcal{F}_{2}\left(\widehat{u}^{2}\right)\right\|_{F_{\ell}} \leq C_{\mathcal{F}}(\mu)\left\|\widehat{u}^{1}-\widehat{u}^{2}\right\|_{E_{\ell}} \\
& \left\|\mathcal{F}_{3}\left(\widehat{u}^{1}, \widehat{p}^{1}\right)-\mathcal{F}_{3}\left(\widehat{u}^{2}, \widehat{p}^{2}\right)\right\|_{F_{\ell}} \leq C_{\mathcal{F}}(\mu)\left\|\widehat{p}^{1}-\widehat{p}^{2}\right\|_{P_{\ell}} .
\end{aligned}
$$

Proof. To estimate $\mathcal{F}_{1}$, with Lemma 5.6, we notice that $\left\|\frac{\partial^{2} Y_{k}}{\partial x_{j}^{2}}\right\|_{D_{\ell}} \leq K_{2}(\mu)$, and we have $\left\|\frac{\partial \widehat{u}}{\partial y_{k}}\right\|_{H^{1+\ell, 1 / 2+\ell / 2}\left(Q_{f}^{\infty}\right)} \leq \mu$. Thus the estimate of $\mathcal{F}_{1}$ follows from Lemma A.3.

To estimate $\mathcal{F}_{2}$, with Lemma 5.6, we notice that $\left\|\frac{\partial Y_{i}}{\partial x_{j}}\right\|_{D_{\ell}} \leq K_{2}(\mu)$, and we have $\left\|\frac{\partial^{2} \widetilde{u}}{\partial y_{i} \partial y_{k}}\right\|_{F_{\ell}} \leq \mu$. Thus the estimate of $\mathcal{F}_{2}$ follows from Lemma 5.6.

To estimate $\mathcal{F}_{3}$, with Lemma 5.6, we notice that $\left\|I-J_{Y}^{T}\right\|_{S_{\ell}} \leq K_{2}(\mu)$, and we have $\|\nabla \widehat{p}\|_{F_{\ell}} \leq \mu$. Thus the estimate of $\mathcal{F}_{3}$ follows from Lemma A.2.

The Lipschitz properties can be proved in the same way.

\subsection{Lipschitz estimates on $\mathcal{G}$, and $\mathrm{g}$}

Recall that

$$
\mathcal{G}(\widehat{u})=e^{-\omega t} \nabla \widehat{u}:\left(I-\operatorname{det}\left(J_{\widehat{X}}\right) J_{\widehat{Y}}^{T}\right), \quad \mathbf{g}(\widehat{u})=e^{-\omega t}\left(I-\operatorname{det}\left(J_{\widehat{X}}\right) J_{\widehat{Y}}\right) \widehat{u},
$$

where

$$
\begin{aligned}
& \widehat{u} \in L^{2}\left(0, \infty ; H^{2+\ell}\left(\Omega_{f}\right)\right) \cap H^{1+\ell / 2}\left(0, \infty ; L^{2}\left(\Omega_{f}\right)\right) \subset H^{1 / 2}\left(0, \infty ; H^{1+\ell}\left(\Omega_{f}\right)\right), \\
& \nabla \widehat{u} \in L^{2}\left(0, \infty ; H^{1+\ell}\left(\Omega_{f}\right)\right) \cap H^{1 / 2+\ell / 2}\left(0, \infty ; L^{2}\left(\Omega_{f}\right)\right), \\
& \operatorname{det}\left(J_{\widehat{X}}\right) J_{\widehat{Y}} \in S_{\ell} .
\end{aligned}
$$

We set

$$
\delta(\widehat{u})=e^{-\omega t}\left|\Gamma_{s}\right|^{-1} \int_{\Gamma_{s}} \mathbf{g}(\widehat{u}) \cdot n, \mathrm{~d} x .
$$

Lemma 5.7. There exists a polynomial $C_{\mathcal{G}} \in \mathcal{P}_{0}^{+}$such that, for all $\mu>0$ satisfying (5.1), all $\widehat{u} \in E_{\ell, \mu}$, all $\widehat{u}^{1} \in E_{\ell, \mu}$, and all $\widehat{u}^{2} \in E_{\ell, \mu}$, we have

$$
\begin{aligned}
\|\mathcal{G}(\widehat{u})\|_{L^{2}\left(0, \infty ; H^{1+\ell}\left(\Omega_{f}\right)\right)} & \leq \mu C_{\mathcal{G}}(\mu), \\
\|\mathcal{G}(\widehat{u})\|_{H^{\ell / 2}\left(0, \infty ; H^{1}\left(\Omega_{f}\right)\right)} & \leq \mu C_{\mathcal{G}}(\mu),
\end{aligned}
$$

and

$$
\begin{aligned}
\left\|\mathcal{G}\left(\widehat{u}^{1}\right)-\mathcal{G}\left(\widehat{u}^{2}\right)\right\|_{L^{2}\left(0, \infty ; H^{1+\ell}\left(\Omega_{f}\right)\right)} & \leq C_{\mathcal{G}}(\mu)\left\|\widehat{u}^{1}-\widehat{u}^{2}\right\|_{E_{\ell}} \\
\left\|\mathcal{G}\left(\widehat{u}^{1}\right)-\mathcal{G}\left(\widehat{u}^{2}\right)\right\|_{H^{\ell / 2}\left(0, \infty ; H^{1}\left(\Omega_{f}\right)\right)} & \leq C_{\mathcal{G}}(\mu)\left\|\widehat{u}^{1}-\widehat{u}^{2}\right\|_{E_{\ell}}
\end{aligned}
$$

Proof. The proof is based on Lemma A.4. 
Step 1. We have

$$
\begin{aligned}
& \|\mathcal{G}(\widehat{u})\|_{L^{2}\left(0, \infty ; H^{1+\ell}\left(\Omega_{f}\right)\right)} \leq C\left\|I-\operatorname{det}\left(J_{\widehat{X}}\right) J_{\widehat{Y}}\right\|_{L^{\infty}\left(0, \infty ; H^{1+\ell}\left(\Omega_{f}\right)\right)}\|\nabla \widehat{u}\|_{L^{2}\left(0, \infty ; H^{1+\ell}\left(\Omega_{f}\right)\right)} \\
& \leq C\|\widehat{u}\|_{E_{\ell}}^{2} .
\end{aligned}
$$

Step 2. Since $\widehat{u}$ belongs to $E_{\ell}, \nabla \widehat{u}$ belongs to $H^{1+\ell, 1 / 2+\ell / 2}\left(Q_{\infty}\right)$. We know that $\left(I-\operatorname{det}\left(J_{\widehat{X}}\right) J_{\widehat{Y}}^{T}\right)$ belongs to $S_{\ell}$. The estimate of $\mathcal{G}(\widehat{u})$ in $H^{\ell / 2}\left(0, \infty ; H^{1}\left(\Omega_{f}\right)\right)$ follows from Lemma A.4.

The Lipschitz estimate for $\mathcal{G}(\widehat{u})$ can be proved in the same way.

Lemma 5.8. There exists a polynomial $C_{\mathbf{g}} \in \mathcal{P}_{0}^{+}$such that, for all $\mu>0$ satisfying (5.1), all $\widehat{u} \in E_{\ell, \mu}$, all $\widehat{u}^{1} \in E_{\ell, \mu}$, and all $\widehat{u}^{2} \in E_{\ell, \mu}$, we have

$$
\|\mathbf{g}(\widehat{u})\|_{H^{1+\ell / 2}\left(0, \infty ; L^{2}\left(\Omega_{f}\right)\right)}+\|\delta(\widehat{u})\|_{H^{1+\ell / 2}(0, \infty)} \leq \mu C_{\mathbf{g}}(\mu),
$$

and

$$
\left\|\mathbf{g}\left(\widehat{u}^{1}\right)-\mathbf{g}\left(\widehat{u}^{2}\right)\right\|_{H^{1+\ell / 2}\left(0, \infty ; L^{2}\left(\Omega_{f}\right)\right)}+\left\|\delta\left(\widehat{u}^{1}\right)-\delta\left(\widehat{u}^{2}\right)\right\|_{H^{1+\ell / 2}(0, \infty)} \leq C_{\mathbf{g}}(\mu)\left\|\widehat{u}^{1}-\widehat{u}^{2}\right\|_{E_{\ell}} .
$$

Proof. We have $\left(I-\operatorname{det}\left(J_{\widehat{X}}\right) J_{\widehat{Y}}\right) \in S_{\ell}$ and $\widehat{u} \in H^{2+\ell, 1+\ell / 2}\left(Q_{f}^{\infty}\right)$. To obtain the estimate of $\mathbf{g}(\widehat{u})$ in $H^{1+\ell / 2}\left(0, \infty ; L^{2}\left(\Omega_{f}\right)\right)$, it is sufficient to apply Lemma A.5. The estimate of $\delta(\widehat{u})$ follows from that of $\mathbf{g}(\widehat{u})$. The Lipschitz estimate can be easily obtained in the same way.

\section{Stabilization of THE NONLINEAR FLUID SYSTEM}

The goal of this section is to prove Theorem 1.1. Our goal is to show that the system

$$
\begin{aligned}
& \frac{\partial \widehat{u}}{\partial t}-\omega \widehat{u}-\nu \Delta \widehat{u}+\nabla \widehat{p}=\widehat{\mathcal{F}}(\widehat{u}, \widehat{p}) \quad \text { and } \quad \operatorname{div} \widehat{u}=\widehat{\mathcal{G}}(\widehat{u})=\operatorname{div} \mathbf{g}(\widehat{u}) \quad \text { in } Q_{F}^{T}, \\
& \widehat{u}=\sum_{i=1}^{N_{c}} f_{i}(t) \xi_{i}(x)+\widehat{\delta}(\widehat{u}) n \quad \text { on } \Sigma_{s}^{\infty}, \quad \widehat{u}=0 \quad \text { on } \Sigma_{0}^{\infty}, \quad \widehat{u}(0)=u_{0} \text { in } \Omega_{f}, \\
& F^{\prime}=\omega F+f, \quad F(0)=0, \\
& f^{\prime}=\omega f+K(P \widehat{u}, F, f) ., \quad f(0)=0, \\
& \widehat{X}(y, t)=y+\int_{0}^{t} e^{-\omega s} \widehat{u}(y, s) \mathrm{d} s, \quad \text { for all } y \in \Omega_{f} \text { and all } t \in[0, \infty), \\
& \Omega_{f}(t)=\widehat{X}\left(\Omega_{f}, t\right), \\
& \widehat{Y}(\widehat{X}(y, t), t)=y, \quad y \in \Omega_{f} \quad \text { and } \quad \widehat{X}(\widehat{Y}(x, t), t)=x, \quad x \in \Omega_{f}(t), t \in[0, \infty),
\end{aligned}
$$

admits a solution $(\widehat{u}, \nabla \widehat{p}, F, f, \widehat{X})$ belonging to $H^{2+\ell, 1+\ell / 2}\left(Q_{f}^{\infty}\right) \times H^{\ell, \ell / 2}\left(Q_{f}^{\infty}\right) \times H^{3+\ell / 2}\left(0, \infty ; \mathbb{R}^{N_{c}}\right) \times$ $H^{2+\ell / 2}\left(0, \infty ; \mathbb{R}^{N_{c}}\right) \times I_{\ell}$, provided that $u_{0} \in H^{1+\ell}\left(\Omega_{f}\right) \cap H_{0}^{1}\left(\Omega_{f}\right)$, with $\operatorname{div} u_{0}=0$, is small enough.

Step 1. We first notice that if $(\widehat{u}, \nabla \widehat{p}, F, f, \widehat{X}) \in H^{2+\ell, 1+\ell / 2}\left(Q_{f}^{\infty}\right) \times H^{\ell, \ell / 2}\left(Q_{f}^{\infty}\right) \times H^{3+\ell / 2}\left(0, \infty ; \mathbb{R}^{N_{c}}\right) \times$ $H^{2+\ell / 2}\left(0, \infty ; \mathbb{R}^{N_{c}}\right) \times I_{\ell}$, is a solution of (6.1), then it is also a solution of (1.7). Indeed, from the condition $\operatorname{div} \widehat{u}=\operatorname{div} \mathbf{g}(\widehat{u})$ and from the definition of $\mathbf{g}(\widehat{u})$, it follows that $\delta(\widehat{u})=0$. Thus, to prove Theorem 1.1, it is sufficient to prove the existence of solution to system (6.1). 
Step 2. We are going to prove the existence of solution to system (6.1) by the Banach fixed point Theorem. For that, for all $\mu>0$ satisfying (5.1), and all $(v, q) \in E_{\ell, \mu} \times P_{\ell, \mu}$, we define $\boldsymbol{\chi}$ and $\Upsilon$ by

$$
\begin{aligned}
& \boldsymbol{\chi}(y, t)=y+\int_{0}^{t} e^{-\omega s} v(y, s) \mathrm{d} s, \quad \text { for all } y \in \Omega_{f} \text { and all } t \in[0, \infty), \\
& \mathbf{\Upsilon}(\boldsymbol{\chi}(y, t), t)=y, \quad y \in \Omega_{f} \quad \text { and } \quad \boldsymbol{\chi}(\boldsymbol{\Upsilon}(x, t), t)=x, \quad x \in \boldsymbol{\chi}\left(\Omega_{f}, t\right), t \in[0, \infty)
\end{aligned}
$$

and the nonlinear terms $\widehat{\mathcal{F}}(v, q), \widehat{\mathcal{G}}(v), \widehat{\mathbf{g}}(v)$, and $\widehat{\delta}(v)$ by

$$
\begin{aligned}
& \widehat{\mathcal{F}}(v, q)=\widehat{\mathcal{F}}_{1}(v)+\widehat{\mathcal{F}}_{2}(v)+\widehat{\mathcal{F}}_{3}(v, q), \\
& \widehat{\mathcal{F}}_{1}(v)=\nu e^{-\omega t} \sum_{j, k} \frac{\partial^{2} \boldsymbol{\Upsilon}_{k}}{\partial x_{j}^{2}}(\chi(y, t), t) \frac{\partial v}{\partial y_{k}}(y, t), \\
& \widehat{\mathcal{F}}_{2}(v)=\nu e^{-\omega t} \sum_{i, j, k} \frac{\partial \boldsymbol{\Upsilon}_{i}}{\partial x_{j}} \frac{\partial \Upsilon_{k}}{\partial x_{j}}(\chi(y, t), t) \frac{\partial^{2} v}{\partial y_{i} \partial y_{k}}(y, t)-\nu e^{-\omega t} \Delta v, \\
& \widehat{\mathcal{F}}_{3}(v, q)=e^{-\omega t}\left(I-J_{\Upsilon}^{T}\right) \nabla q, \\
& \widehat{\mathcal{G}}(v)=e^{-\omega t} \nabla v:\left(I-\operatorname{det}\left(J_{\chi}\right) J_{\Upsilon}^{T}\right), \quad \widehat{\mathbf{g}}(v)=e^{-\omega t}\left(I-\operatorname{det}\left(J_{\chi}\right) J_{\Upsilon}\right) v \\
& \text { and } \widehat{\delta}(v)=e^{-\omega t}\left|\Gamma_{s}\right|^{-1} \int_{\Gamma_{s}} \widehat{\mathbf{g}}(v) \cdot n \mathrm{~d} x .
\end{aligned}
$$

For all $(v, q) \in E_{\ell, \mu} \times P_{\ell, \mu}$, we introduce the system

$$
\begin{aligned}
& \frac{\partial \widetilde{u}}{\partial t}-\omega \widetilde{u}-\nu \Delta \widetilde{u}+\nabla \widetilde{p}=\widehat{\mathcal{F}}(v, q) \quad \text { and } \quad \operatorname{div} \widetilde{u}=\widehat{\mathcal{G}}(v)=\widehat{\mathbf{g}}(v) \quad \text { in } Q_{F}^{T}, \\
& \widetilde{u}=\sum_{i=1}^{N_{c}} f_{i}(t) \xi_{i}(x)+\widehat{\delta}(v) n \text { on } \Sigma_{s}^{\infty}, \quad \widetilde{u}=0 \text { on } \Sigma_{0}^{\infty}, \quad \widetilde{u}(0)=u_{0} \text { in } \Omega_{f}, \\
& F^{\prime}=\omega F+f, \quad F(0)=0, \\
& f^{\prime}=\omega f+K(P \widetilde{u}, F, f), \quad f(0)=0, \\
& \widetilde{X}(y, t)=y+\int_{0}^{t} e^{-\omega s} \widetilde{u}(y, s) \mathrm{d} s, \quad \text { for all } y \in \Omega_{f} \text { and all } t \in[0, \infty), \\
& \Omega_{f}(t)=\widetilde{X}\left(\Omega_{f}, t\right), \\
& \widetilde{Y}(\widetilde{X}(y, t), t)=y, \quad y \in \Omega_{f} \quad \text { and } \quad \widetilde{X}(\widetilde{Y}(x, t), t)=x, \quad x \in \Omega_{f}(t), t \in[0, \infty),
\end{aligned}
$$

We denote by $\mathcal{N}$, the mapping from $E_{\ell, \mu} \times P_{\ell, \mu}$ into $E_{\ell} \times P_{\ell}$, defined by

$$
(v, q) \longmapsto(\widetilde{u}, \widetilde{p}),
$$

where $(\widetilde{u}, \widetilde{p}, f, F, \widetilde{X})$ is the solution to $(6.4)$.

Our goal is to prove that, for $\mu>0$ small enough, $\mathcal{N}$ is a contraction in $E_{\ell, \mu} \times P_{\ell, \mu}$. From Theorem 4.1, and Lemmas 5.7 to 5.9 , it follows that

$$
\|\mathcal{N}(v, q)\|_{E_{\ell} \times P_{\ell}} \leq C_{1}\left(\left\|u_{0}\right\|_{H^{1+\ell}\left(\Omega_{f}\right)}+\mu C_{\mathcal{F}}(\mu)+\mu C_{\mathcal{G}}(\mu)+\mu C_{\mathbf{g}}(\mu)\right)
$$


for all $(v, q) \in E_{\ell, \mu} \times P_{\ell, \mu}$, and

$$
\begin{aligned}
& \left\|\mathcal{N}\left(v_{1}, q_{1}\right)-\mathcal{N}\left(v_{2}, q_{2}\right)\right\|_{E_{\ell} \times P_{\ell}} \\
& \leq C_{1}\left(C_{\mathcal{F}}(\mu)+C_{\mathcal{G}}(\mu)+C_{\mathbf{g}}(\mu)\right)\left\|\left(v_{1}, q_{1}\right)-\left(v_{2}, q_{2}\right)\right\|_{E_{\ell} \times P_{\ell}}
\end{aligned}
$$

for all $\left(v_{1}, q_{1}\right) \in E_{\ell, \mu} \times P_{\ell, \mu}$ and all $\left(v_{2}, q_{2}\right) \in E_{\ell, \mu} \times P_{\ell, \mu}$. We choose $\mu>0$ such that

$$
C_{1}\left(C_{\mathcal{F}}(\mu)+C_{\mathcal{G}}(\mu)+C_{\mathbf{g}}(\mu)\right) \leq \frac{1}{2},
$$

and we choose $u_{0}$ such that

$$
C_{1}\left\|u_{0}\right\|_{H^{1+\ell}} \leq \frac{\mu}{2}
$$

Since $(0,0) \in E_{\ell, \mu} \times P_{\ell, \mu}$, the space $E_{\ell, \mu} \times P_{\ell, \mu}$ is nonempty. From (6.5), (6.7), and (6.8), it follows that $\mathcal{N}$ is a mapping from $E_{\ell, \mu} \times P_{\ell, \mu}$ into itself, and with (6.6) and (6.7), it follows that $\mathcal{N}$ is a contraction in $E_{\ell, \mu} \times P_{\ell, \mu}$. Therefore $\mathcal{N}$ admits a fixed point which is a solution to (6.1). Due to Step 1, the proof of Theorem 1.1 is complete.

\section{Appendix A. Product estimates}

Lemma A.1. If $f \in S_{\ell}$ and $g \in S_{\ell}$, then $f g \in S_{\ell}$.

Proof. If $f \in L^{\infty}\left(0, \infty ; H^{1+\ell}\left(\Omega_{f}\right)\right)$ and $g \in L^{\infty}\left(0, \infty ; H^{1+\ell}\left(\Omega_{f}\right)\right)$, then

$$
\|f g\|_{L^{\infty}\left(0, \infty ; H^{1+\ell}\left(\Omega_{f}\right)\right)} \leq K_{m}\|f\|_{L^{\infty}\left(0, \infty ; H^{1+\ell}\left(\Omega_{f}\right)\right)}\|g\|_{L^{\infty}\left(0, \infty ; H^{1+\ell}\left(\Omega_{f}\right)\right)},
$$

where $K_{m}$ is the continuity constant of the bilinear mapping $m$ from $H^{1+\ell}\left(\Omega_{f}\right) \times H^{1+\ell}\left(\Omega_{f}\right)$ into $H^{1+\ell}\left(\Omega_{f}\right)$ defined by $m(f, g)=f g$.

To estimate $\partial_{t}(f g)$ in $L^{2}\left(0, \infty ; H^{1 / 2+\ell / 2}\left(\Omega_{f}\right)\right) \cap H^{1 / 2+\ell / 2}\left(0, \infty ; L^{2}\left(\Omega_{f}\right)\right)$, we write

$$
\partial_{t}(f g)=\left(\partial_{t} f\right) g+f\left(\partial_{t} g\right)
$$

The estimates of the two terms are similar. Let us look at the first term. The estimate of $\left(\partial_{t} f\right) g$ in $L^{2}\left(0, \infty ; H^{1 / 2+\ell / 2}\left(\Omega_{f}\right)\right)$ is clear:

$$
\left\|\left(\partial_{t} f\right) g\right\|_{L^{2}\left(0, \infty ; H^{1+\ell}\left(\Omega_{f}\right)\right)} \leq K_{m}\left\|\partial_{t} f\right\|_{L^{2}\left(0, \infty ; H^{1+\ell}\left(\Omega_{f}\right)\right)}\|g\|_{L^{\infty}\left(0, \infty ; H^{1+\ell}\left(\Omega_{f}\right)\right)} .
$$

We have to estimate the seminorm $\left|\left(\partial_{t} f\right) g\right|_{H^{\ell / 2}\left(0, \infty ; L^{2}\left(\Omega_{f}\right)\right)}$. We treat the cases $1<\ell<\frac{3}{2}, \frac{1}{2}<\ell<1$ and $\ell=1$ separately.

Step 1. We first treat the case when $1<\ell<\frac{3}{2}$. Let us estimate the time derivative of $\left(\partial_{t} f\right) g$. We have

$$
\partial_{t}\left(\left(\partial_{t} f\right) g\right)=\left(\partial_{t t}^{2} f\right) g+\left(\partial_{t} f\right)\left(\partial_{t} g\right)
$$

We estimate separately the two terms $\left(\partial_{t t}^{2} f\right) g$ and $\left(\partial_{t} f\right)\left(\partial_{t} g\right)$ in $H^{\ell / 2-1 / 2}\left(0, \infty ; L^{2}\left(\Omega_{f}\right)\right)$. For the second term, we have

$$
\left\|\left(\partial_{t} f\right)\left(\partial_{t} g\right)\right\|_{H^{\ell / 2-1 / 2}\left(0, \infty ; L^{2}\left(\Omega_{f}\right)\right)} \leq C\left\|\partial_{t} f\right\|_{H^{\ell / 2}\left(0, \infty ; H^{1}\left(\Omega_{f}\right)\right)}\left\|\partial_{t} g\right\|_{H^{\ell / 2}\left(0, \infty ; H^{1}\left(\Omega_{f}\right)\right)} .
$$


For the first term, we have

$$
\left\|\left(\partial_{t t}^{2} f\right) g\right\|_{L^{2}\left(0, \infty ; L^{2}\left(\Omega_{f}\right)\right)} \leq C\left\|\partial_{t t}^{2} f\right\|_{L^{2}\left(0, \infty ; H^{\ell-1}\left(\Omega_{f}\right)\right)}\|g\|_{L^{\infty}\left(0, \infty ; H^{1+\ell}\left(\Omega_{f}\right)\right)} .
$$

We estimate the semi-norm $\left|\left(\partial_{t t}^{2} f\right) g\right|_{H^{\ell / 2-1 / 2}\left(0, \infty ; L^{2}\left(\Omega_{f}\right)\right)}$ by difference quotients

$$
\begin{aligned}
& \left|\left(\partial_{t t}^{2} f\right) g\right|_{H^{\ell / 2-1 / 2}\left(0, \infty ; L^{2}\left(\Omega_{f}\right)\right)}^{2} \\
& =\int_{0}^{\infty} \int_{0}^{\infty} \frac{\left\|\partial_{t t}^{2} f(s) g(s)-\partial_{t t}^{2} f(\tau) g(\tau)\right\|_{L^{2}\left(\Omega_{f}\right)}^{2}}{|s-\tau|^{\ell}} \\
& \leq \int_{0}^{\infty} \int_{0}^{\infty} \frac{\left\|\partial_{t t}^{2} f(s)(g(s)-g(\tau))\right\|_{L^{2}\left(\Omega_{f}\right)}^{2}}{|s-\tau|^{\ell}} \int_{0}^{\infty} \int_{0}^{\infty} \frac{\left\|\left(\partial_{t t}^{2} f(s)-\partial_{t t}^{2} f(\tau)\right) g(\tau)\right\|_{L^{2}\left(\Omega_{f}\right)}^{2}}{|s-\tau|^{\ell}} \\
& \leq \iint_{|s-\tau|<1} \frac{\left\|\partial_{t t}^{2} f(s)(g(s)-g(\tau))\right\|_{L^{2}\left(\Omega_{f}\right)}^{2}}{|s-\tau|^{\ell}} \iint_{|s-\tau|>1} \frac{\left\|\partial_{t t}^{2} f(s)(g(s)-g(\tau))\right\|_{L^{2}\left(\Omega_{f}\right)}^{2}}{|s-\tau|^{\ell}} \\
& \quad+C\left|\partial_{t t}^{2} f\right|_{H^{\ell / 2-1 / 2}\left(0, \infty ; L^{2}\left(\Omega_{f}\right)\right)}^{2}\|g\|_{L^{\infty}\left(0, \infty ; H^{1+\ell}\left(\Omega_{f}\right)\right)}^{2} .
\end{aligned}
$$

To estimate the first integral, we write

$$
\begin{aligned}
& \iint_{|s-\tau|<1} \frac{\left\|\partial_{t t}^{2} f(s)(g(s)-g(\tau))\right\|_{L^{2}\left(\Omega_{f}\right)}^{2}}{|s-\tau|^{\ell}} \\
& \leq \iint_{|s-\tau|<1}\left\|\left(\int_{0}^{1} \partial_{t} g(\tau+\theta(s-\tau))\right) d \theta\right\|_{L^{\infty}\left(\Omega_{f}\right)}^{2}\left\|\partial_{t t}^{2} f(s)\right\|_{L^{2}\left(\Omega_{f}\right)}^{2} \frac{1}{|s-\tau|^{\ell-2}} \mathrm{~d} s \mathrm{~d} \tau \\
& \leq C\left\|\partial_{t} g\right\|_{L^{\infty}\left(0, \infty ; H^{\ell}\left(\Omega_{f}\right)\right)}^{2} \int_{0}^{1} \frac{\mathrm{d} r}{|r|^{\ell-2}} \int_{0}^{\infty}\left\|\partial_{t t}^{2} f(s)\right\|_{L^{2}\left(\Omega_{f}\right)}^{2} \mathrm{~d} s \\
& \leq C\left\|\partial_{t t}^{2} f\right\|_{L^{2}\left(0, \infty ; H^{\ell-2}\left(\Omega_{f}\right)\right)}^{2}\left\|\partial_{t} g\right\|_{L^{\infty}\left(0, \infty ; H^{\ell}\left(\Omega_{f}\right)\right)}^{2} .
\end{aligned}
$$

For the second term, we can write

$$
\begin{aligned}
& \iint_{|s-\tau|>1} \frac{\left\|\partial_{t t}^{2} f(s)(g(s)-g(\tau))\right\|_{L^{2}\left(\Omega_{f}\right)}^{2}}{|s-\tau|^{\ell}} \mathrm{d} \mathrm{d} \tau \\
& \leq C\|g\|_{L^{\infty}\left(0, \infty ; H^{1+\ell}\left(\Omega_{f}\right)\right)}^{2} \iint_{\{\tau-s>1\} \cup\{s-\tau>1\}} \frac{\left\|\partial_{t t}^{2} f(s)\right\|_{L^{2}\left(\Omega_{f}\right)}^{2}}{|s-\tau|^{\ell}} \mathrm{d} s \mathrm{~d} \tau \\
& \leq C\|g\|_{L^{\infty}\left(0, \infty ; H^{1+\ell}\left(\Omega_{f}\right)\right)}^{2} \int_{1}^{\infty} \frac{\mathrm{d} r}{|r|^{\ell}} \int_{0}^{\infty}\left\|\partial_{t t}^{2} f(s)\right\|_{L^{2}\left(\Omega_{f}\right)}^{2} \mathrm{~d} s \\
& \leq C\left\|\partial_{t t}^{2} f\right\|_{L^{2}\left(0, \infty ; L^{2}\left(\Omega_{f}\right)\right)}^{2}\|g\|_{L^{\infty}\left(0, \infty ; H^{1+\ell}\left(\Omega_{f}\right)\right)}^{2} \\
& \leq C\|f\|_{S_{\ell}}^{2}\|g\|_{S_{\ell}}^{2} .
\end{aligned}
$$


Step 2. We now treat the case when $\frac{1}{2}<\ell<1$. We estimate the semi-norm $\left|\left(\partial_{t} f\right) g\right|_{H^{1 / 2+\ell / 2}\left(0, \infty ; L^{2}\left(\Omega_{f}\right)\right)}$ by difference quotients. We have

$$
\begin{aligned}
& \left|\left(\partial_{t} f\right) g\right|_{H^{\ell / 2+1 / 2}\left(0, \infty ; L^{2}\left(\Omega_{f}\right)\right)}^{2} \\
& =\int_{0}^{\infty} \int_{0}^{\infty} \frac{\left\|\partial_{t} f(s) g(s)-\partial_{t} f(\tau) g(\tau)\right\|_{L^{2}\left(\Omega_{f}\right)}^{2}}{|s-\tau|^{\ell+2}} \\
& \leq \int_{0}^{\infty} \int_{0}^{\infty} \frac{\left\|\partial_{t} f(s)(g(s)-g(\tau))\right\|_{L^{2}\left(\Omega_{f}\right)}^{2}}{|s-\tau|^{\ell+2}} \int_{0}^{\infty} \int_{0}^{\infty} \frac{\left\|\left(\partial_{t} f(s)-\partial_{t} f(\tau)\right) g(\tau)\right\|_{L^{2}\left(\Omega_{f}\right)}^{2}}{|s-\tau|^{\ell+2}} \\
& \leq \iint_{|s-\tau|<1} \frac{\left\|\partial_{t} f(s)(g(s)-g(\tau))\right\|_{L^{2}\left(\Omega_{f}\right)}^{2}}{|s-\tau|^{\ell+2}} \iint_{|s-\tau|>1} \frac{\left\|\partial_{t} f(s)(g(s)-g(\tau))\right\|_{L^{2}\left(\Omega_{f}\right)}^{2}}{|s-\tau|^{\ell+2}} \\
& \quad+C\left|\partial_{t} f\right|_{H^{\ell / 2+1 / 2}\left(0, \infty ; L^{2}\left(\Omega_{f}\right)\right)}^{2}\|g\|_{L^{\infty}\left(0, \infty ; H^{1+\ell}\left(\Omega_{f}\right)\right)}^{2} .
\end{aligned}
$$

To estimate the first integral, we write

$$
\begin{aligned}
& \iint_{|s-\tau|<1} \frac{\left\|\partial_{t} f(s)(g(s)-g(\tau))\right\|_{L^{2}\left(\Omega_{f}\right)}^{2}}{|s-\tau|^{\ell+2}} \\
& \leq \iint_{|s-\tau|<1}\left\|\left(\int_{0}^{1} \partial_{t} g(\tau+\theta(s-\tau))\right) d \theta\right\|_{H^{\ell}\left(\Omega_{f}\right)}^{2}\left\|\partial_{t} f(s)\right\|_{H^{1}\left(\Omega_{f}\right)}^{2} \frac{1}{|s-\tau|^{\ell}} \mathrm{d} s \mathrm{~d} \tau \\
& \leq C\left\|\partial_{t} g\right\|_{L^{\infty}\left(0, \infty ; H^{\ell}\left(\Omega_{f}\right)\right)}^{2} \int_{0}^{1} \frac{\mathrm{d} r}{|r|^{\ell}} \int_{0}^{\infty}\left\|\partial_{t} f(s)\right\|_{H^{1}\left(\Omega_{f}\right)}^{2} \mathrm{~d} s \\
& \leq C\left\|\partial_{t} f\right\|_{L^{2}\left(0, \infty ; H^{1}\left(\Omega_{f}\right)\right)}^{2}\left\|\partial_{t} g\right\|_{L^{\infty}\left(0, \infty ; H^{\ell}\left(\Omega_{f}\right)\right)}^{2} .
\end{aligned}
$$

For the second term, we can write

$$
\begin{aligned}
& \iint_{|s-\tau|>1} \frac{\left\|\partial_{t} f(s)(g(s)-g(\tau))\right\|_{L^{2}\left(\Omega_{f}\right)}^{2}}{|s-\tau|^{\ell+2}} \mathrm{~d} \mathrm{~d} \tau \\
& \leq C\|g\|_{L^{\infty}\left(0, \infty ; H^{1+\ell}\left(\Omega_{f}\right)\right)}^{2} \iint_{\{|s-\tau|>1\}} \frac{\left\|\partial_{t} f(s)\right\|_{L^{2}\left(\Omega_{f}\right)}^{2}}{|s-\tau|^{\ell+2}} \mathrm{~d} s \mathrm{~d} \tau \\
& \leq C\|g\|_{L^{\infty}\left(0, \infty ; H^{1+\ell}\left(\Omega_{f}\right)\right)}^{2} \int_{1}^{\infty} \frac{\mathrm{d} r}{|r|^{\ell+2}} \int_{0}^{\infty}\left\|\partial_{t} f(s)\right\|_{L^{2}\left(\Omega_{f}\right)}^{2} \mathrm{~d} s \\
& \leq C\left\|\partial_{t} f\right\|_{L^{2}\left(0, \infty ; L^{2}\left(\Omega_{f}\right)\right)}^{2}\|g\|_{L^{\infty}\left(0, \infty ; H^{1+\ell}\left(\Omega_{f}\right)\right)}^{2} \\
& \leq C\|f\|_{S_{\ell}}^{2}\|g\|_{S_{\ell}}^{2} .
\end{aligned}
$$

Step 3. We now treat the case when $\ell=1$. We have already estimated $\left(\partial_{t} f\right) g$ in $L^{2}\left(0, \infty ; L^{2}\left(\Omega_{f}\right)\right)$. To estimate $\partial_{t}\left(\left(\partial_{t} f\right) g\right)$ in $L^{2}\left(0, \infty ; L^{2}\left(\Omega_{f}\right)\right)$, we write

$$
\partial_{t}\left(\left(\partial_{t} f\right) g\right)=\partial_{t t}^{2} f g+\left(\partial_{t} f\right) \partial_{t} g
$$

We have

$$
\left\|\partial_{t t}^{2} f g\right\|_{L^{2}\left(0, \infty ; L^{2}\left(\Omega_{f}\right)\right)} \leq\left\|\partial_{t t}^{2} f\right\|_{L^{2}\left(0, \infty ; H^{1+\ell}\left(\Omega_{f}\right)\right)}\|g\|_{L^{\infty}\left(0, \infty ; H^{1+\ell}\left(\Omega_{f}\right)\right)}
$$


and

$$
\left\|\partial_{t} f \partial_{t} g\right\|_{L^{2}\left(0, \infty ; L^{2}\left(\Omega_{f}\right)\right)} \leq\left\|\partial_{t} f\right\|_{L^{2}\left(0, \infty ; H^{1+\ell}\left(\Omega_{f}\right)\right)}\left\|\partial_{t} g\right\|_{L^{\infty}\left(0, \infty ; H^{\ell}\left(\Omega_{f}\right)\right)} .
$$

The proof is complete.

Lemma A.2. If $f \in S_{\ell}$ and $g \in H^{\ell, \ell / 2}\left(Q_{f}^{\infty}\right)$, then $f g \in H^{\ell, \ell / 2}\left(Q_{f}^{\infty}\right)$.

Proof. The estimate of $f g$ in $L^{2}\left(0, \infty ; H^{\ell}\left(\Omega_{f}\right)\right)$ is easy. Indeed, we have

$$
\|f g\|_{L^{2}\left(0, \infty ; H^{\ell}\left(\Omega_{f}\right)\right)} \leq C\|f\|_{L^{\infty}\left(0, \infty ; H^{1+\ell}\left(\Omega_{f}\right)\right)}\|g\|_{L^{2}\left(0, \infty ; H^{\ell}\left(\Omega_{f}\right)\right)} .
$$

To prove that $f g$ belongs to $H^{\ell / 2}\left(0, \infty ; L^{2}\left(\Omega_{f}\right)\right)$, we treat only the case when $1<\ell<\frac{3}{2}$. The cases when $\frac{1}{2}<\ell<1$ and $\ell=1$ can be treated with obvious modifications as we did in the proof of Lemma A.1. To estimate the semi-norm $|f g|_{H^{\ell / 2}\left(0, \infty ; L^{2}\left(\Omega_{f}\right)\right)}$, we use difference quotients. We have

$$
\begin{aligned}
& |f g|_{H^{\ell / 2}\left(0, \infty ; L^{2}\left(\Omega_{f}\right)\right)}^{2} \\
& =\int_{0}^{\infty} \int_{0}^{\infty} \frac{\left\|f(s) g(s)-f(\tau) \partial_{t} g(\tau)\right\|_{L^{2}\left(\Omega_{f}\right)}^{2}}{|s-\tau|^{\ell+1}} \mathrm{~d} \tau \\
& \leq \int_{0}^{\infty} \int_{0}^{\infty} \frac{\|f(s)(g(s)-g(\tau))\|_{L^{2}\left(\Omega_{f}\right)}^{2}}{|s-\tau|^{\ell+1}} \mathrm{~d} \tau+\int_{0}^{\infty} \int_{0}^{\infty} \frac{\|(f(s)-f(\tau)) g(\tau)\|_{L^{2}\left(\Omega_{f}\right)}^{2}}{|s-\tau|^{\ell+1}} \mathrm{~d} \tau \\
& \leq C\|f\|_{L^{\infty}\left(0, \infty ; H^{1+\ell}\left(\Omega_{f}\right)\right)}^{2}\|g\|_{H^{\ell / 2}\left(0, \infty ; L^{2}\left(\Omega_{f}\right)\right)}^{2}+\iint_{|s-\tau|<1} \frac{\|(f(s)-f(\tau)) g(\tau)\|_{L^{2}\left(\Omega_{f}\right)}^{2}}{|s-\tau|^{\ell+1}} \mathrm{~d} \tau \\
& +\iint_{|s-\tau|>1} \frac{\|(f(s)-f(\tau)) g(\tau)\|_{L^{2}\left(\Omega_{f}\right)}^{2}}{|s-\tau|^{\ell+1}} \mathrm{~d} s \mathrm{~d} \tau .
\end{aligned}
$$

To estimate the first integral, we write

$$
\begin{aligned}
& \iint_{|s-\tau|<1} \frac{\|(f(s)-f(\tau)) g(\tau)\|_{L^{2}\left(\Omega_{f}\right)}^{2}}{|s-\tau|^{\ell+1}} \mathrm{~d} \mathrm{~d} \tau \\
& \leq C \iint_{|s-\tau|<1}\left\|\left(\int_{0}^{1} \partial_{t} f(\tau+\theta(s-\tau))\right) d \theta\right\|_{H^{\ell}\left(\Omega_{f}\right)}^{2}|s-\tau|^{1-\ell}\|g(\tau)\|_{L^{2}\left(\Omega_{f}\right)}^{2} \mathrm{~d} s \mathrm{~d} \tau \\
& \leq C\left\|\partial_{t} f\right\|_{L^{\infty}\left(0, \infty ; H^{\ell}\left(\Omega_{f}\right)\right)}^{2}\|g\|_{H^{\ell / 2}\left(0, \infty ; L^{2}\left(\Omega_{f}\right)\right)}^{2} \leq C\|f\|_{S_{\ell}}^{2}\|g\|_{H^{\ell / 2}\left(0, \infty ; L^{2}\left(\Omega_{f}\right)\right)}^{2} .
\end{aligned}
$$

For the second term, we can write

$$
\begin{aligned}
& \iint_{|s-\tau|>1} \frac{\|(f(s)-f(\tau)) g(\tau)\|_{L^{2}\left(\Omega_{f}\right)}^{2}}{|s-\tau|^{\ell+1}} \\
& \leq C\|f\|_{L^{\infty}\left(0, \infty ; H^{1+\ell}\left(\Omega_{f}\right)\right)}^{2} \int_{1}^{\infty} \frac{\mathrm{d} r}{|r|^{\ell+1}} \int_{0}^{\infty}\|g(\tau)\|_{L^{2}\left(\Omega_{f}\right)}^{2} \mathrm{~d} \tau \\
& \leq C\|f\|_{L^{\infty}\left(0, \infty ; H^{1+\ell}\left(\Omega_{f}\right)\right)}^{2}\|g\|_{L^{2}\left(0, \infty ; L^{2}\left(\Omega_{f}\right)\right)}^{2} \\
& \leq C\|f\|_{L^{\infty}\left(0, \infty ; H^{1+\ell}\left(\Omega_{f}\right)\right)}^{2}\|g\|_{H^{\ell, \ell / 2}\left(Q_{f}^{\infty}\right)}^{2}
\end{aligned}
$$


The proof is complete.

Lemma A.3. If $f \in D_{\ell}$ and $g \in H^{1+\ell, 1 / 2+\ell / 2}\left(Q_{f}^{\infty}\right)$, then $f g \in H^{\ell, \ell / 2}\left(Q_{f}^{\infty}\right)$.

Proof. The estimate of $f g$ in $L^{2}\left(0, \infty ; H^{\ell}\left(\Omega_{f}\right)\right)$ is easy. Indeed, we have

$$
\|f g\|_{L^{2}\left(0, \infty ; H^{\ell}\left(\Omega_{f}\right)\right)} \leq C\|f\|_{L^{\infty}\left(0, \infty ; H^{\ell}\left(\Omega_{f}\right)\right)}\|g\|_{L^{2}\left(0, \infty ; H^{1+\ell}\left(\Omega_{f}\right)\right)} .
$$

To prove that $f g$ belongs to $H^{\ell / 2}\left(0, \infty ; L^{2}\left(\Omega_{f}\right)\right)$, we treat only the case when $1<\ell<\frac{3}{2}$. The cases when $\frac{1}{2}<\ell<1$ and $\ell=1$ can be treated with obvious modifications. To estimate $f g$ in $H^{\ell / 2}\left(0, \infty ; L^{2}\left(\Omega_{f}\right)\right)$ when $1<\ell<\frac{3}{2}$, we can use difference quotients and adapt the proof of Lemma A.2.

Lemma A.4. If $f \in S_{\ell}$ and $g \in H^{1+\ell, 1 / 2+\ell / 2}\left(Q_{f}^{\infty}\right)$, then $f g \in L^{2}\left(0, \infty ; H^{1+\ell}\left(\Omega_{f}\right)\right)$ $\cap H^{\ell / 2}\left(0, \infty ; H^{1}\left(\Omega_{f}\right)\right)$.

Proof. The estimate of $f g$ in $L^{2}\left(0, \infty ; H^{1+\ell}\left(\Omega_{f}\right)\right)$ is easy. Indeed, we have

$$
\|f g\|_{L^{2}\left(0, \infty ; H^{1+\ell}\left(\Omega_{f}\right)\right)} \leq C\|f\|_{L^{\infty}\left(0, \infty ; H^{1+\ell}\left(\Omega_{f}\right)\right)}\|g\|_{L^{2}\left(0, \infty ; H^{1+\ell}\left(\Omega_{f}\right)\right)} .
$$

To estimate $f g$ in $H^{\ell / 2}\left(0, \infty ; H^{1}\left(\Omega_{f}\right)\right)$, we can use difference quotients and adapt the proof of Lemma A.2.

Lemma A.5. If $f \in S_{\ell}$ and $g \in H^{2+\ell, 1+\ell / 2}\left(Q_{f}^{\infty}\right)$, then $f g \in L^{2}\left(0, \infty ; H^{1+\ell}\left(\Omega_{f}\right)\right)$ $\cap H^{1+\ell / 2}\left(0, \infty ; L^{2}\left(\Omega_{f}\right)\right)$.

Proof. The estimate of $f g$ in $L^{2}\left(0, \infty ; H^{1+\ell}\left(\Omega_{f}\right)\right)$ is easy. Indeed, we have

$$
\|f g\|_{L^{2}\left(0, \infty ; H^{1+\ell}\left(\Omega_{f}\right)\right)} \leq C\|f\|_{L^{\infty}\left(0, \infty ; H^{1+\ell}\left(\Omega_{f}\right)\right)}\|g\|_{L^{2}\left(0, \infty ; H^{1+\ell}\left(\Omega_{f}\right)\right)} .
$$

To estimate $f g$ in $H^{1+\ell / 2}\left(0, \infty ; L^{2}\left(\Omega_{f}\right)\right)$, we estimate $\partial_{t} f g$ and $f \partial_{t} g$ in $H^{\ell / 2}\left(0, \infty ; L^{2}\left(\Omega_{f}\right)\right)$. The first term is estimated by writing

$$
\left\|\partial_{t} f g\right\|_{H^{\ell / 2}\left(0, \infty ; L^{2}\left(\Omega_{f}\right)\right)} \leq\left\|\partial_{t} f\right\|_{H^{\ell / 2}\left(0, \infty ; H^{1}\left(\Omega_{f}\right)\right)}\|g\|_{H^{\ell / 2}\left(0, \infty ; H^{2}\left(\Omega_{f}\right)\right)} .
$$

For the second term, we have $\partial_{t} g \in H^{\ell, \ell / 2}\left(Q_{f}^{\infty}\right)$, and $f \in S_{\ell}$. We apply Lemma A.2.

\section{REFERENCES}

[1] J.T. Beale, The initial value problem for the Navier-Stokes equations with a free surface. Commun. Pure Appl. Math. 34 (1981) 359-392.

[2] A. Bensoussan, G. Da Prato, M.C. Delfour and S.K. Mitter, Representation and Control of Infinite Dimensional Systems, Second edition, Birkhäuser (2006).

[3] M. Boulakia, S. Guerrero and T. Takahashi, Well-posedness for the coupling between a viscous incompressible fluid and an elastic structure. Nonlinearity 32 (2019) 3548-3592.

[4] D. Coutand and S. Shkoller, Motion of an elastic solid inside an incompressible viscous fluid. Arch. Ratl. Mech. Anal. 176 (2005) 25-102.

[5] P. Cumsille and T. Takahashi, Wellposedness for the system modeling the motion of a rigid body of arbitrary form in an incompressible viscous fluid. Czechoslovak Math. J. 58 (2008) 961-992.

[6] S. Ervedoza and E. Zuazua, A systematic method for building smooth controls for smooth data. Discr. Continu. Dyn. Syst. B 14 (2010) 1375-1401.

[7] M. Fournié, M. Ndiaye and J.-P. Raymond, Feedback stabilization of a two-dimensional fluid-structure interaction system with mixed boundary conditions. SIAM J. Control Optim. 57 (2019) 3322-3359.

[8] G. Grubb and V.A. Solonnikov, Boundary value problems for the nonstationary Navier-Stokes equations treated by pseudodifferential methods. Math. Scand. 69 (1991) 217-290. 
[9] J. Haubner, M. Ulbrich and S. Ulbrich, Analysis of shape optimization problems for unsteady fluid-structure interaction. Inverse Problems 36 (2020) 034001.

[10] J. Haubner, Shape optimization for fluid-structure interaction, Ph.D. Thesis, TUM, Germany (2020).

[11] M. Hieber and M. Murata, The Lp-approach to the fluid-rigid body interaction problem for compressible fluids. Evolut. Equ. Control Theory 4 (2015) 69-87.

[12] M. Ignatova, I. Kukavica, I. Lasiecka and A. Tuffaha, On well-posedness for a free boundary fluid-structure model. J. Math. Phys. 53 (2012) 115624-1-13.

[13] M. Ignatova, I. Kukavica, I. Lasiecka and A. Tuffaha, On well-posedness and small data global existence for an interface damped free boundary fluid-structure model. Nonlinearity 27 (2014) 467-499.

[14] I. Kukavica, A. Tuffaha and M. Ziane, Strong solutions to a Navier-Stokes-Lamé system on a domain with a non-flat boundary. Nonlinearity 24 (2011) 159-176.

[15] I. Kukavica and A. Tuffaha, Solutions to a fluid-structure interaction free boundary problem. Discrete Cont. Dyn. Syst. 32 (2012) 1355-1389.

[16] I. Kukavica and A. Tuffaha, Regularity of solutions to a free boundary problem of fluid-structure interaction. Indiana Univ. Math. J. 61 (2012) 1817-1859.

[17] J.-L. Lions and E. Magenes, Problèmes aux limites non homogènes, Vol. 2, Dunod, Paris (1968).

[18] I. Lasiecka and Y. Lu, Interface feedback control stabilization of a nonlinear fluid-structure interaction. Nonlinear Anal. 75 (2012) 1449-1460.

[19] D. Maity, J.-P. Raymond and A. Roy, Maximal-in-time existence of strong solutions of a 3D fluid structure interaction model. SIAM J. Math. Anal. 52 (2020) 6338-6378.

[20] P.A. Nguyen and J.-P. Raymond, Boundary stabilization of the Navier-Stokes equations in the case of mixed boundary conditions. SIAM J. Control Optim. 53 (2015) 3006-3039.

[21] J.-P. Raymond, Feedback stabilization of a fluid-structure model. SIAM J. Control Optim. 48 (2010) 5398-5443.

[22] J.-P. Raymond, Feedback boundary stabilization of the three-dimensional incompressible Navier-Stokes equations. J. Math. Pures Appl. 87 (2007) 627-669.

[23] J.-P. Raymond, Stokes and Navier-Stokes equations with a nonhomogeneous divergence condition. Discrete Continu. Dyn. Syst. B 14 (2010) 1537-1564.

[24] J.-P. Raymond and L. Thevenet, Boundary feedback stabilization of the two dimensional Navier-Stokes equations with finite dimensional controllers. Discr. Continu. Dyn. Syst. A 27 (2010) 1159-1187.

[25] J.-P. Raymond and M. Vanninathan, A fluid-structure model coupling the Navier-Stokes equations and the Lamé system. $J$. Math. Pures Appl. 102 (2014) 546-596.

[26] J.-P. Raymond, Stabilizability of infinite-dimensional systems by finite-dimensional controls. Comput. Methods Appl. Math. 19 (2019) 797-811.

[27] V.A. Solonnikov, Solvability of the problem of evolution of a viscous incompressible fluid bounded by a free surface on a finite time interval. St. Petersburg Math. J. 3 (1992) 189-220.

[28] T. Takahashi, M. Tucsnak and G. Weiss, Stabilization of a fluid-rigid body system. J. Differ. Equ. 259 (2015) $6459-6493$.

[29] R. Temam, Navier-Stokes equations. Theory and numerical analysis, Reprint of the 1984 edition. AMS Chelsea Publishing, Providence, RI (2001).

[30] R. Temam, Navier-Stokes equations and nonlinear functional analysis, Second edition. CBMS-NSF Regional Conference Series in Applied Mathematics, 66. Society for Industrial and Applied Mathematics (SIAM), Philadelphia, PA (1995).

[31] G. Troianiello, Elliptic differential equations and obstacle problems. The University Series in Mathematics. Plenum Press, New York (1987).

[32] X. Zhang and E. Zuazua, Long-time behavior of a coupled heat-wave system arising in fluid-structure interaction. Arch. Ratl. Mech. Anal. 184 (2007) 49-120.

[33] X. Zhang and E. Zuazua, Asymptotic behavior of a hyperbolic-parabolic coupled system arising in fluid-structure interaction. Free Boundary Problem. Boarding school. Ser. Number. Math. 154. Birkhäuser, Basel (2007) 445-455. 